\title{
Visual mechanisms for voice-identity recognition flexibly adjust to auditory noise level
}

\author{
Corrina Maguinness ${ }^{1,2 *} \&$ Katharina von Kriegstein ${ }^{1,2}$ \\ 1 Chair of Cognitive and Clinical Neuroscience, Technische Universität Dresden, Dresden, Germany \\ 2 Max Planck Institute for Human Cognitive and Brain Sciences, Leipzig, Germany \\ *corrina.maguinness@tu-dresden.de; katharina.von_kriegstein@tu-dresden.de \\ *Corresponding author
}

\begin{abstract}
Recognising the identity of voices is a key ingredient of communication. Visual mechanisms support this ability: recognition is better for voices previously learned with their corresponding face (compared to a control condition). This so-called 'face-benefit' is supported by the fusiform face area (FFA), a region sensitive to facial form and identity. Behavioural findings indicate that the facebenefit increases in noisy listening conditions. The neural mechanisms for this increase are unknown. Here, using functional magnetic resonance imaging, we examined responses in facesensitive regions while participants recognised the identity of auditory-only speakers (previously learned by face) in high (SNR -4 dB) and low (SNR $4 \mathrm{~dB}$ ) levels of auditory noise. We observed a facebenefit in both noise levels, for most participants (16 of 21). In high-noise, the recognition of facelearned speakers engaged the right posterior superior temporal sulcus motion-sensitive face area (pSTS-mFA), a region implicated in the processing of dynamic facial cues. The face-benefit in highnoise also correlated positively with increased functional connectivity between this region and voicesensitive regions in the temporal lobe in the group of 16 participants with a behavioural face-benefit. In low-noise, the face-benefit was robustly associated with increased responses in the FFA and to a lesser extent the right pSTS-mFA. The findings highlight the remarkably adaptive nature of the visual network supporting voice-identity recognition in auditory-only listening conditions.
\end{abstract}

Keywords: multisensory, audio-visual, voice-identity, motion, FFA, pSTS, predictive coding

\section{Introduction}

Human communication is often based on input from more than one sensory modality. For example, when we listen to someone's voice, we are often concurrently exposed to their face. These audio-visual correspondences make communication more robust. For instance, in noisy listening conditions, observers can more accurately perceive what someone says when they can also view their accompanying lip-movements (Sumby and Pollack, 1954; 
Erber, 1969; Rosenblum, Johnson and Saldana, 1996; Ross et al., 2007). Perhaps surprisingly, these visual mechanisms are also engaged under auditory-only listening conditions (Sheffert and Olson, 2004; von Kriegstein and Giraud, 2006; von Kriegstein et al., 2008): Listeners are more accurate at recognising the identity of a speaker by their voice alone, when that speaker has been previously learned by face, in comparison to a control condition (Sheffert and Olson, 2004; von Kriegstein et al., 2008; Schall et al., 2013; Schelinski, Riedel and von Kriegstein, 2014; Zäske, Mühl and Schweinberger, 2015). This behavioural enhancement, termed the "face-benefit", emerges rapidly following approximately two minutes of audio-visual experience with the speaker's identity (von Kriegstein et al., 2008). The face-benefit is observed in the majority of neurotypical participants (i.e., 76\% - von Kriegstein et al., 2008; Maguinness, Schall and von Kriegstein, 2021) and might be one of the reasons why most of us recognise familiar voices with such ease (Sidtis and Kreiman, 2012; Maguinness and von Kriegstein, 2017; Maguinness, Roswandowitz and von Kriegstein, 2018; Stevenage, 2018; Lavan et al., 2019).

Audio-visual learning likely benefits unisensory processing as the information in each sensory stream is governed by a common cause (for reviews see Shams and Seitz, 2008; von Kriegstein, 2012). Voices are caused by physical visual structures (i.e., the vocal tract) and provide information about the visual characteristics of the speaker. For example, fundamental frequency (i.e., pitch), formant frequencies and vocal-tract resonance (i.e., timbre) map well to, and are predictive of structural form cues, including face-identity (Krauss, Freyberg and Morsella, 2002; Ives, Smith and Patterson, 2005; Smith and Patterson, 2005; Smith et al., 2005; Ghazanfar et al., 2007; Mavica and Barenholtz, 2013; Smith et al., 2016; Smith et al., 2016; Kim et al., 2019; Oh et al., 2019). This non-arbitrary coupling of sensory information is reflected at the neural level: The face-benefit for auditory-only voice-identity recognition has been shown to be mediated by responses in the fusiform face area (FFA) (von Kriegstein and Giraud, 2006; von Kriegstein et al., 2008; Schall et al., 2013). The FFA is a visual face-sensitive region (Kanwisher, McDermott and Chun, 1997) implicated in the processing of structural facial form (i.e., the invariant static features of the face) and face-identity (Eger, Schyns and Kleinschmidt, 2004; Grill-Spector, Knouf and Kanwisher, 2004; Rotshtein et al., 2005; Kanwisher and Yovel, 2006; Ewbank and Andrews, 2008; Xu et al., 2009; Liu, Harris and Kanwisher, 2010; Schiltz et al., 2010; Axelrod and Yovel, 2015; Weibert and Andrews, 2015). Responses in this region, during voice-identity compared to speech recognition, occur as early as 110 milliseconds after auditory onset (Schall et al., 2013) - a time point when voice-identity recognition has yet to be achieved (Schweinberger, 2001; Schweinberger, Kloth and Robertson, 2011). This quick response is thought to be mediated by direct connections between the FFA and voicesensitive regions in the superior temporal gyrus and sulcus (STG/S) (von Kriegstein et al., 2005; von Kriegstein and Giraud, 2006; Blank, Anwander and von Kriegstein, 2011; Hölig et al., 2014a, 2014b; Schall and von Kriegstein, 2014). 
What might be a governing principle for these cross-modal interactions during auditory tasks? One proposal offered by an audio-visual model of human auditory communication (von Kriegstein et al., 2005, 2008; von Kriegstein and Giraud, 2006; review see von Kriegstein, 2012) is that visual mechanisms assist subsequent auditory recognition by generating predictions about, and thus placing constraints on the sensory processing of, the incoming auditory signal (von Kriegstein et al., 2008; Kiebel, Daunizeau and Friston, 2009; Blank, Kiebel and von Kriegstein, 2015). Such a process would be particularly beneficial for optimising voice-identity recognition when the auditory signal is weak or degraded, with predictions assisting recognition by "filling in" missing sensory information. In agreement with this suggestion, recent behavioural evidence shows that, in individuals who display a face-benefit, the face-benefit increases with decreasing signal-to-noise ratios (SNR) of the auditory signal (Maguinness, Schall and von Kriegstein, 2021). This indicates that visual mechanisms may help to systematically resolve incoming noisy auditory input. While previous studies have shown a positive relationship between increased FFA responses and the face-benefit in relatively clear listening conditions (von Kriegstein et al., 2008), to-date, it is unclear whether the face-benefit for voice-identity processing in noise is also facilitated by responses in the FFA.

Voice-identity recognition is mediated by the extraction of relatively invariant 'static' voice features, such as fundamental frequency and vocal tract resonances (Voiers, 1964; Lavner, Rosenhouse and Gath, 2001; Latinus and Belin, 2011). However, there is also evidence that dynamic articulatory idiosyncrasies, such as speech rhythm (Van Lancker, Kreiman and Emmorey, 1985; Van Lancker, Kreiman and Wickens, 1985; Leemann, Kolly and Dellwo, 2014; Dellwo, Leemann and Kolly, 2015; He and Dellwo, 2016) and formant dynamics (Ingram, Prandolini and Ong, 1996; Mc Dougall, 2004, 2006; Mc Dougall and Nolan, 2007; Zuo and Mok, 2015) play a role. These dynamic cues can support voice-identity recognition when other cues such as fundamental frequency are unreliable (Fellowes, Remez and Rubin, 1997; Remez, Fellowes and Rubin, 1997; Sheffert et al., 2002; Zuo and Mok, 2015; Simmons et al., 2020). In parallel, similar adaptive mechanisms have also been observed to support face-identity recognition when static form cues are degraded. In challenging viewing conditions, facial motion cues or "dynamic facial signatures" (O'Toole, Roark and Abdi, 2002; Roark et al., 2003) can provide a complementary route to recognition (Knight and Johnston, 1997; Lander, Christie and Bruce, 1999; Lander and Bruce, 2000; Lander and Chuang, 2005; Longmore and Tree, 2013; Dobs, Bülthoff and Schultz, 2016). These cues are likely processed by motion-sensitive regions of the face-network (O'Toole, Roark and Abdi, 2002; Bernstein and Yovel, 2015; Girges, Spencer and O'Brien, 2015; Girges, O'Brien and Spencer, 2016) i.e., the posterior superior temporal sulcus motion-sensitive face area or pSTS-mFA (Fox, Iaria and Barton, 2009; Schultz and Pilz, 2009; Pitcher et al., 2011; Bernstein et al., 2018). Like static cues, dynamic spatio-temporal cues in the face and voice share common source identity information (Kamachi et al., 2003; Lachs and Pisoni, 2004; Mc Dougall, 2006; Smith et al., 2016; Simmons et al., 2020). Thus, in conditions with 
noise, the face-benefit for voice-identity recognition might rely on complementary dynamic face-identity cues processed in the pSTS-mFA, rather than the FFA. Such a finding would indicate that stored visual cues may be used in an adaptable manner, in line with the nature of the auditory input, to support voice-identity processing (Figure 1).
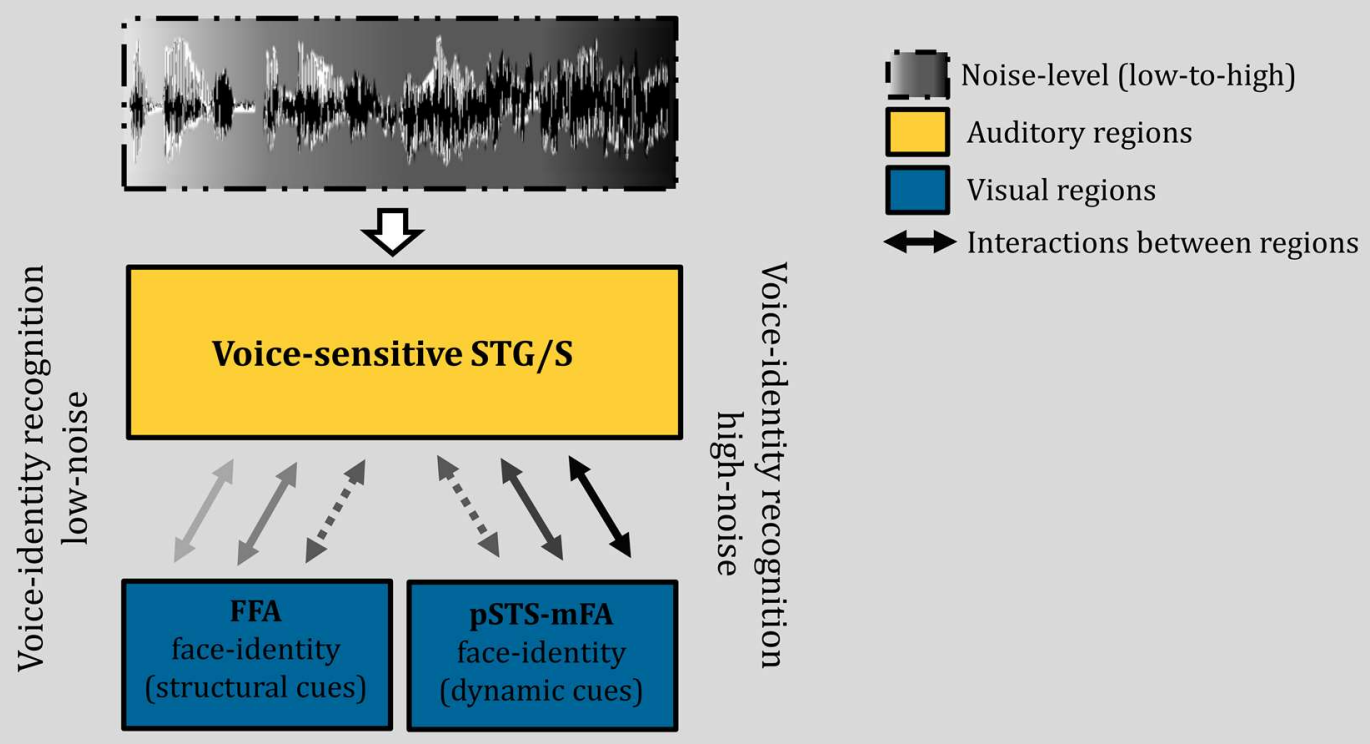

Figure 1. Schematic overview of potential audio-visual interactions between voice- (yellow) and face-sensitive (blue) brain regions, during auditory-only voice-identity processing. The interactions may vary as a function of the noise level present in the auditory signal (top panel lowto-high noise, light grey-to-black transitioning colours). Interactions between regions are indicated via bidirectional arrows. The arrow colours (light grey-to-black) reflect the noise level (low-to-high) in the auditory signal. Bold arrows depict potential strong interactions between brain regions, dashed arrows depict weaker interactions. Voice-identity recognition low-noise. Voice-identity recognition is supported by interactions between the FFA, a region sensitive to structural faceidentity cues, and the anterior and mid voice-sensitive STG/S (von Kriegstein and Giraud, 2006; Schall and von Kriegstein, 2014). To date, this has been documented for relatively low-noise listening conditions e.g., MRI-scanner noise, or in conditions with a positive signal-to-noise ratio (bold light grey/grey arrows, left of figure). The FFA may also support voice-identity processing in increasingly noisy listening conditions, however, the region may be less recruited as static vocal cues become increasingly degraded (dashed grey arrow, left of figure). Voice-identity recognition high-noise. The pSTS-mFA may be involved in voice-identity recognition in increasingly noisy listening conditions. Potentially, interactions between the pSTS-mFA, a region sensitive to dynamic face cues, and voice-sensitive regions in the anterior and mid STG/S, may be observed. This may be particularly apparent in high-noise levels (bold dark grey/black arrows, right of figure), and less so in lower noise levels (dashed grey arrow, right of figure). 
The aim of the present study was to investigate the visual mechanisms underpinning the face-benefit for voice-identity recognition in noisy listening conditions (Figure 1). We used functional magnetic resonance imaging (fMRI) to examine responses in both the FFA and the pSTS-mFA while participants engaged in an auditory-only voiceidentity recognition task in two levels of auditory noise: high-noise (signal-to-noise ratio -4 $\mathrm{dB}$ ) and low-noise (signal-to-noise ratio $+4 \mathrm{~dB}$ ). All speakers had been learned before MRIdata acquisition. Crucially, half of the speakers had been learned by seeing and listening to videos of the speaker talking (voice-face learning), while the other half had been learned by listening to the speaker while viewing a visual control stimulus depicting the speaker's occupation (voice-occupation learning). Thus, the design was a 2 × 2 factorial design with the factors noise-level (high-noise, low-noise) and learning condition (voice-face, voiceoccupation). Our first and central aim was to test whether in the high-noise listening condition (in contrast to the lower noise condition) there are increased responses in the FFA, the pSTS-mFA, or both for face-learned (in comparison to occupation-learned) speakers, i.e., a noise-level x learning condition interaction. Second, we expected a positive correlation between listeners' face-benefit scores and responses in visual face-sensitive regions, i.e., a behaviourally relevant relationship with neural responses. Third, we expected that face-sensitive regions underpinning the face-benefit in noise would share functional connections with voice-sensitive regions in the STG/S (Figure 1). On the behavioural level, we expected based on previous findings (Sheffert and Olson, 2004; for review see von Kriegstein, 2012), that across both noise levels, speakers learned by face would be more accurately recognised than those learned by occupation and that this facebenefit would be greatest in the high-noise condition (Maguinness, Schall and von Kriegstein, 2021).

\section{Materials and Methods}

\section{Participants}

Twenty-three neurotypical German speaking adults (12 female; mean age 25 years, SD 2.9 years), recruited from the Max Planck Institute for Cognitive and Brain Sciences participant database, took part in this study. All were right handed (Oldfield, 1971) and reported normal hearing and normal vision. All participants gave their informed written consent prior to participation according to the procedures approved by the Ethics Committee of the Medical Faculty at the University of Leipzig (299-12-24092012). Two subjects were excluded from neuroimaging and behavioural analysis owing to below chance performance on the voice-identity recognition task inside the MRI-machine. Analysis for the fMRI and behavioural task data was based on twenty-one participants (12 female; mean age 25 years, SD 3 years). Since one additional participant did not complete the face area 
localiser runs (see below), analysis of the fMRI functional face area localiser data was based on twenty participants.

Stimuli

Audio-visual Training and Auditory-only Voice-identity Recognition Test

The stimuli for the audio-visual voice-face and voice-occupation training sessions comprised of ten audio-visual and five auditory recordings of fourteen five- to six-word sentences, from six male German speakers (22-27 years old). All sentences were semantically neutral (e.g., "Die Ente kommen an das Ufer" English: "The ducks come to the shore"). Audio-visual stimuli for the voice-face training were video sequences, which displayed the talking face of the speaker. For the audio-visual voice-occupation training the speaker's face was replaced with an image depicting the speaker's occupation. Both audiovisual sequences were presented for the same duration. The stimuli for the auditory-only voice-identity recognition test (in the MRI-scanner) consisted of 30 two-word sentences, presented in noise. Each sentence started with "Er" (English: "He") and finished with a verb (e.g., "Er beisst", English: "He bites"). All sentences were spoken by the same six male speakers presented during the audio-visual training phase.

The audio-visual training and test stimuli were recorded using a high-definition camera (Legria HF S10 HD-Camcorder, Canon, Japan) and an external condenser microphone [TLM 50 (Neumann, Berlin, Germany); Mic-Preamp, Mic-Amp F-35 (Lake People, Konstanz, Germany); soundcard, Power Mac G5 (Apple Inc., CA, USA); Sound Studio 3 (Felt Tip, Inc. NY, USA) (44.1 kHz sampling rate and 16 bit resolution)]. For the audiovisual training, video stimuli were edited in Final Cut Pro software (Apple Inc., CA, USA) to include a circular mask, which excluded the background while revealing the face of the speaker. Videos were cropped to 727 x 545 pixels. In addition, for each speaker a single frame depicting the speaker in a neutral pose was extracted from the video sequence. Three symbols representing an occupation (painter, chef, and mechanic) were taken from Clip Art (http://office.microsoft.com/en-us/). The auditory stimuli were adjusted for overall mean amplitude using Matlab7 (MathWorks, MA, USA). For the auditory-only test, stimuli were masked with pink noise (created in Matlab7 by filtering Gaussian white noise). Pink noise was chosen as it has similar spectral qualities to speech and has been used in previous studies examining the face-benefit on auditory processing (e.g., Schall et al., 2013; Maguinness, Schall and von Kriegstein, 2021) and audio-visual speech-in-noise processing (Ross et al., 2007; Riedel et al., 2015). Unlike white noise, it also has a stronger power in the frequency range $(100-250 \mathrm{~Hz})$ which is sensitive to spectral components of the speech signal that support identity-recognition i.e., fundamental frequency (F0) (Traunmüller and Eriksson, 1994; Pernet and Belin, 2012). The stimuli were mixed with noise of varying intensities to produce signal-to-noise ratios (SNR) of $-4 \mathrm{~dB}$ (referred to hereafter as 'high- 
noise') and $+4 \mathrm{~dB}$ (referred to hereafter as 'low-noise'). The noise was ramped and introduced with a linear 50 millisecond fade-in and fade-out. For four participants included in the neuroimaging and behavioural analyses the auditory stimuli in the high-noise condition had an SNR of $-8 \mathrm{~dB}$ and the low-noise $+4 \mathrm{~dB}$. Example auditory stimuli can be viewed in Figure 2. This was subsequently adjusted to SNR $-4 \mathrm{~dB} /+4 \mathrm{~dB}$ in order to improve behavioural task performance inside the MRI-machine. These four participants could reliably complete the task (i.e., above chance performance). See Supporting Information Figure 1, for individual behavioural data. As we were interested in the within-subject effect of noise-level (i.e., relative effect of noise), all 21 participants were included in the analysis.

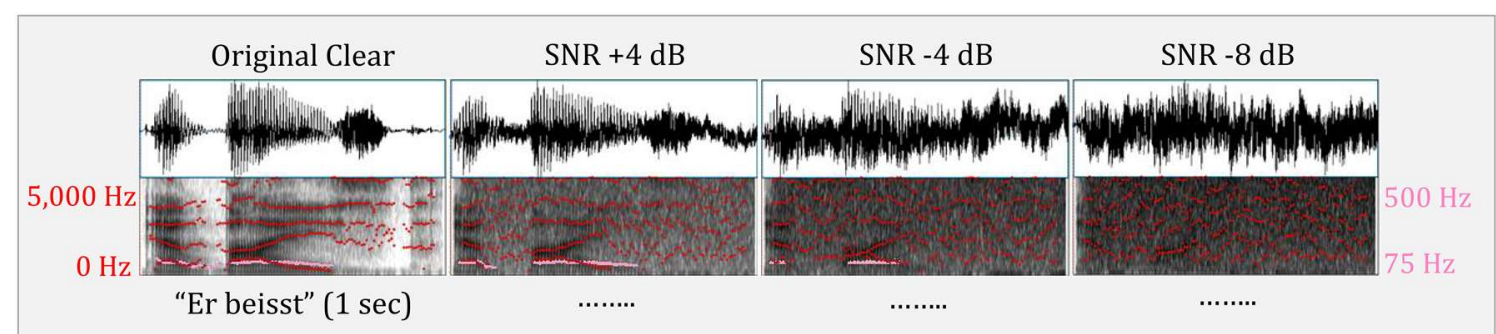

Figure 2. Example of the stimuli from the auditory-only voice-identity recognition task. Stimuli for one sample sentence, "Er beisst" ("he bites"), are shown for signal-to-noise ratios of $+4 \mathrm{~dB},-4 \mathrm{~dB}$, and $-8 \mathrm{~dB}$. The original clear audio file is shown on the left for comparison purposes. The spectrogram (lower panel) displays the fundamental frequency (F0), i.e., pitch of the voice, in pink and the formant frequencies (F1-F5) in red.

\section{Visual-only Face Area Localiser}

We used a functional localiser (design as von Kriegstein et al., 2008; Borowiak, Maguinness and von Kriegstein, 2019) to establish the location of the face-sensitive FFA and the pSTS-mFA within participants. The face stimuli consisted of still frames, extracted using Final Cut Pro software (Apple Inc., CA, USA), from video sequences of 50 identities (25 female; 19-34 years). All identities were unfamiliar and had no overlap with the identities from the main experiment. The video sequences were recorded using a digital video camera (HD-Camcorder LEGRIA HSF100; Canon Inc., Tokyo, Japan). In each sequence, the person was asked to stand still and look into the camera (frontal face view) with a neutral expression. In addition, each person articulated the letters of the German alphabet, maintaining the neutral pose. The object stimuli were static images of 50 different common objects, which were taken from the database of object images described in (von Kriegstein et al., 2008). All images were presented in colour and cropped to measure 768 x 576 pixels.

\section{Experimental Procedure}

All experiments including the audio-visual training, auditory-only voice-identity recognition test, and visual-only face area localiser were run using Presentation (www.neurobs.com) software. 


\section{Audio-visual Training (Before MRI-data Acquisition)}

Prior to MRI data acquisition, participants were familiarised with six male speakers using an established audio-visual training paradigm which has been shown to elicit the facebenefit and associated responses in the FFA (see von Kriegstein et al., 2008; Schall et al., 2013). As we were specifically interested in the effect of noise level on these responses, we kept the learning design as comparable as possible to the previous studies. Three of the speakers were learned through an audio-visual sequence which displayed the corresponding dynamic facial identity of the speaker (i.e., video). The other three speakers were learned through an audio-visual control sequence, which displayed a visual image of the occupation of the speaker (Figure 3A). The inclusion of an audio-visual, rather than an auditory-only, control condition ensured that participants were always exposed to personrelated visual information during learning. We refer to the two audio-visual training conditions as voice-face learning and voice-occupation learning, respectively. The three speakers assigned to the voice-face learning or the voice-occupation learning conditions were counterbalanced across participants. In both conditions the participant also learned the name of the speaker.

Familiarisation with the speakers was achieved through two training rounds. Each training round consisted of a learning stage, followed by an evaluation stage. In the learning stage, participants were exposed to a series of trials which first displayed the name of the speaker ( 1 second), immediately followed by an audio-track (approx. 2 seconds). The audiotrack in each trial consisted of one of ten five- to six-word sentences (e.g., "Die Enten kommen an das Ufer" English: "The ducks come to the shore"). In the voice-face learning condition the audio-track was accompanied by the corresponding time synchronised video track. In the voice-occupation learning condition it was accompanied by the occupation symbol of the respective speaker. The voice-face or voice-occupation trials were presented in two separate blocks. There were 20 trials per speaker in each block i.e., each speaker was heard uttering each five- to six-word sentence twice (i.e., 20 trials $\times 3$ speakers), with a total of 60 trials per voice-face or voice-occupation block. In each block, the initial 15 trials were presented grouped by a speaker-identity i.e., 5 trials were presented consecutively per speaker-identity. In the remaining 45 trials, all speaker-identities were presented in a randomised order. In total, there were 120 trials for a learning stage (60 voice-face trials, 60 voice-occupation trials). At the end of the learning stage, participants then completed an evaluation stage. In each evaluation trial, participants heard the voice of a speaker (auditory-only), which was immediately followed by a name (half of trials) or a static face/occupation image (half of trials) presented onscreen. The auditory clips consisted of four novel five- to six-word sentences which were not contained within the learning stage. In the whole evaluation stage, each speaker uttered each five- to six-word sentence twice, so that there were 8 evaluation trials per speaker (i.e., 24 trials for the voice-face and 24 trials for the voice-occupation condition, totalling 48 trials for an evaluation stage). On half 
of the trials, for each learning condition, the voice-identity matched the name or static face/occupation image, while the other half of the trials contained mismatched names and images. The mismatching names and images were always taken from the same speaker set (i.e., voice-face or voice-occupation). Participants indicated via button-press whether the voice-identity matched the name or face/occupation image ("yes" or "no"). The name or image remained onscreen until a response was made. Participants received feedback in the form of the correct name and voice-face/voice-occupation combination. All participants reached the learning criterion of $\geq 80$ percent correct (von Kriegstein et al., 2008) after two rounds of training, indicating that they could reliably match the correct combinations of voice, name and face/occupation.

\section{Auditory-only Voice-identity Recognition Test (fMRI)}

In the auditory-only voice-identity recognition test (Figure 3B), participants listened to two-word sentences (e.g., "Er liest", English: "He reads"). The sentences were uttered by the 6 speakers which participants had been familiarised with during the audiovisual training phase. In each trial, a sentence (1 second duration) was followed by a visual name presented onscreen (1 second duration) (Figure 3C). When the visual name was displayed, the participant was instructed to respond 'yes' or 'no' as to whether the name matched the identity of the heard speaker. Participants indicated their response by pressing one of two assigned buttons with their right hand (responses were made within the 1 second name display interval). Half of the trials contained speakers who had been previously learned by face in the audio-visual training phase (voice-face learning), while the other half contained speakers who had been learned with an occupation symbol (voiceoccupation). In addition, half of the trials were presented in high-noise and half in low-noise listening conditions. Thus, the experiment was a $2 \times 2$ factorial design with the factors learning (voice-face, voice-occupation) and noise-level (high-noise, low-noise). The experimental trials were blocked by condition type: (i) voice-face high-noise; (ii) voice-face low-noise; (iii) voice-occupation high-noise; (iv) voice-occupation low-noise. Each block contained 9 trials ( 18 seconds). There were 20 blocks per condition ( 720 trials in total). The blocks were presented in a randomised order and were interleaved with baseline silence blocks (14 seconds), in which participants looked at a fixation cross. The blocks were presented over four 11-minute runs, with 20 task blocks per run. 


\section{A. audio-visual training (before MRI-acquisition)}

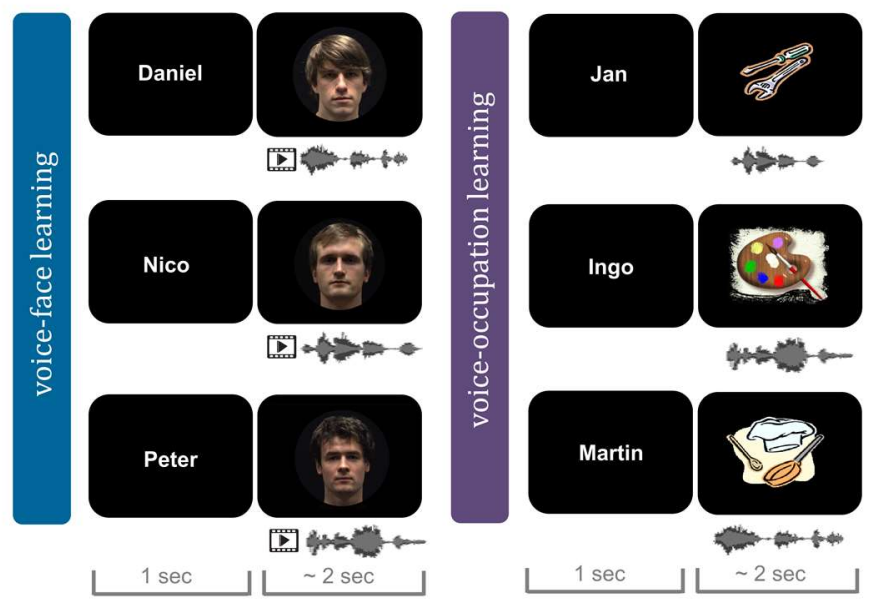

\section{B. auditory-only voice-identity recognition (fMRI)}

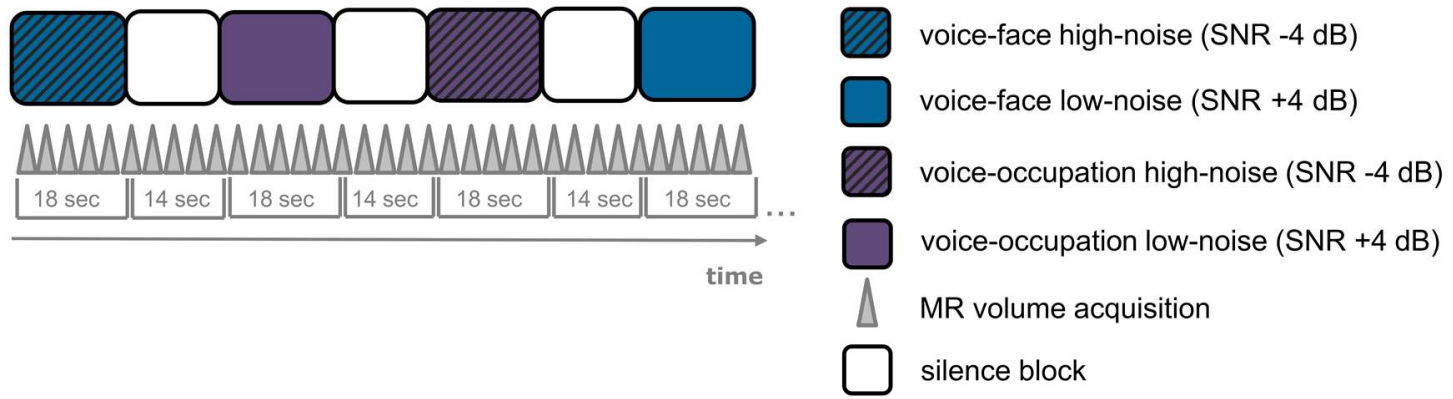

C. sample trials: auditory-only voice-identity recognition (fMRI)

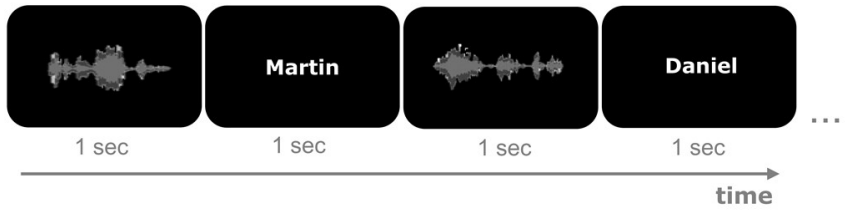

Figure 3. A schematic illustration of the audio-visual training phase and the auditory-only voice-identity recognition test. (A) Audio-visual training. Prior to MRI-acquisition, participants learned the voice and name of six speakers. Half of the speakers were learned in conjunction with their corresponding face i.e., video (voice-face learning) and the other half with an occupation symbol (voice-occupation learning). The speakers assigned to each learning condition were counterbalanced across participants. Each speaker was learned for approximately 2 minutes in total. (B) Auditoryonly voice-identity recognition (fMRI). During MRI-acquisition, participants listened to auditoryonly sentences spoken by the familiarised six speakers in high- and low-noise listening conditions. The speakers were presented in separate blocks, blocked by learning type (voice-face or voiceoccupation) and noise level. Blocks were presented in a randomised order and interleaved with silence baseline blocks. Functional MR images were acquired continuously. (C) Sample trials: auditory-only voice-identity recognition (fMRI). On each trial, participants heard a speaker utter 
a sentence, followed by the presentation of a speaker's name onscreen. Participants decided whether the name matched the identity of the preceding voice.

\section{face area ROI localiser}
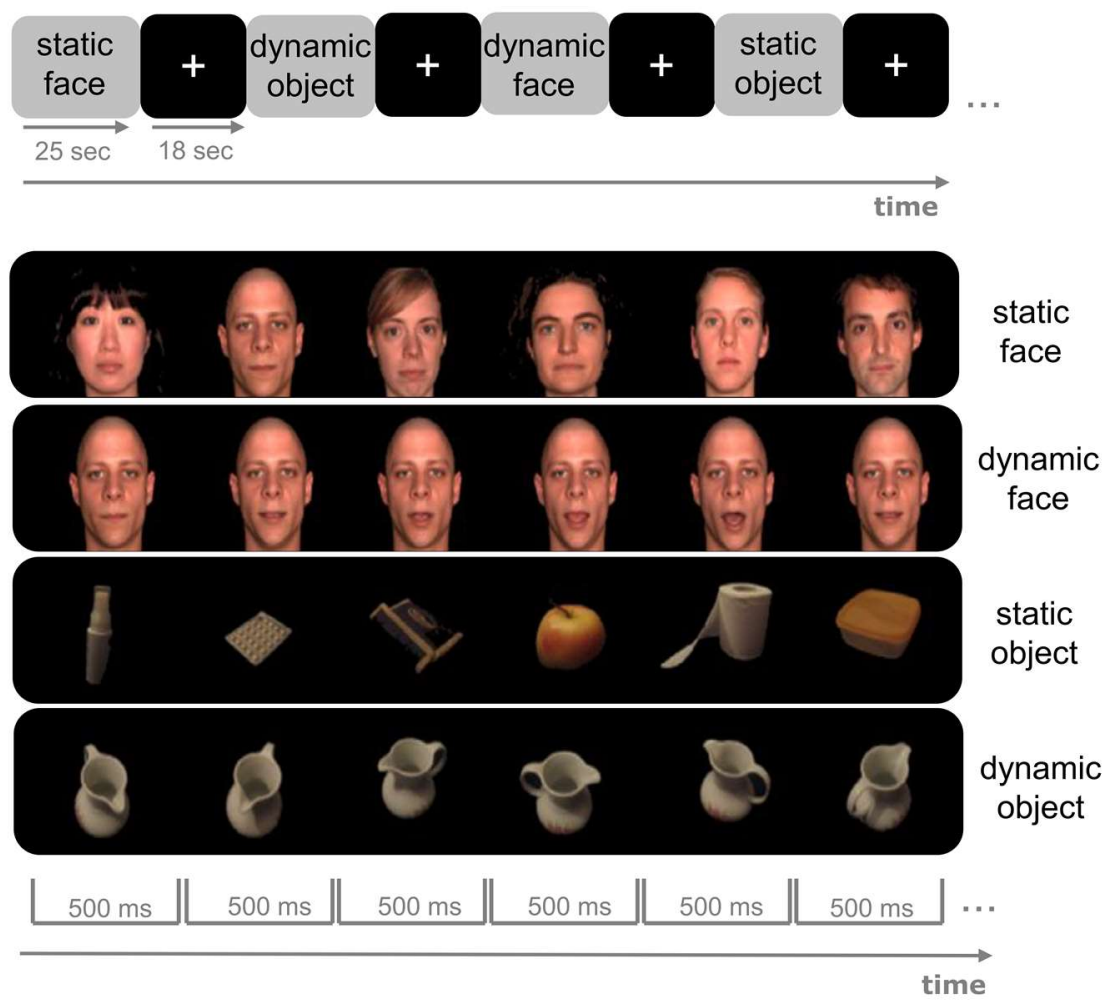

Figure 4. Visual face area ROI localiser. Images of faces and objects were shown in separate blocks, interleaved with baseline blocks displaying a fixation cross. There were four block types: static face, dynamic face, static object, and dynamic object. Participants were asked to attend to the images presented onscreen.

\section{Visual Face Area Localiser (fMRI)}

A standard experiment which contrasted responses during the viewing of faces versus objects was used to localise the FFA and the pSTS-mFA (von Kriegstein et al., 2008; Pitcher et al., 2011; Borowiak, Maguinness and von Kriegstein, 2019). Participants were presented with still frames taken from videos of faces or objects under four different conditions: i) images of faces from different identities, with different facial speech poses; ii) images of faces from the same identity, with different facial speech poses; iii) images of different objects, from different viewpoints; iv) images of the same object, from different viewpoints. All images were static in nature and presented onscreen for 500 milliseconds with no inter-stimulus interval. The quick succession rate of image presentation induced an implied motion effect for images of the same facial identity (dynamic face) and for images of the same object (dynamic object). This implied motion effect was not apparent when 
images depicted different facial identities (static face) or different objects (static object). See Figure 4 for illustration of the four conditions. Images were blocked by condition type and each block contained 50 images. There were four blocks per condition type and each block lasted 25 seconds. The blocks were presented in a randomised order over two 6-minute runs ( 8 blocks per run) and interleaved with baseline blocks, where a fixation cross was presented for 18 seconds. Participants were asked to attentively view the images within each block.

Image Acquisition

\section{Functional MRI}

Functional images for the auditory-only voice-identity recognition task and the visual face area localiser were acquired on a 3T Siemens Prisma MR scanner (Siemens, Erlangen, Germany), equipped with a 20-channel head coil. Images were acquired using a gradient-echo echo planar imaging continuous scanning sequence (TE 30ms, flip angle 90 degrees, TR 2.64 s, 42 slices, whole-brain coverage, acquisition bandwidth $128 \mathrm{kHz}$ (2004 $\mathrm{Hz} / \mathrm{pix}$ ), $2.5 \mathrm{~mm}$ slice thickness, $0.5 \mathrm{~mm}$ inter slice gap, in plane resolution $3 \mathrm{x} 3 \mathrm{~mm}$, ascending interleaved slice acquisition). Geometric distortions were characterised by a B0 field map. The field map scan consisted of a pair of 2D gradient echo images with different echo times (TE1/TE2 $=4.58 \mathrm{~ms} / 7.04 \mathrm{~ms}$ ). This field map was acquired once per participant before the first experimental task run began. All images were acquired in AC-PC orientation. 980 volumes were acquired for the auditory-only voice-identity recognition task $(245 \times 4$ runs) and 262 volumes for the visual face area localiser (131 x 2 runs).

\section{Structural MRI}

Structural images for each participant were attained from the MPI brain database. The images had been acquired on either the same 3T Siemens Prisma scanner used for functional image acquisition, or a 3T Siemens-Tim Trio, Magnetom Verio, or Numaris 4 scanner. Images were acquired using either a T1-weighted three-dimensional magnetization-prepared rapid gradient echo (MP-RAGE) sequence or a magnetization-prepared 2 rapid gradient echo (MP2-RAGE) sequence (MP-RAGE: $n=$ 13; MP2-RAGE: $n=8$ ). Imaging parameters for the MP-RAGE sequence were TR $=2300$ $\mathrm{ms}, \mathrm{TE}=2.98 \mathrm{~ms}, \mathrm{TI}=900 \mathrm{~ms}$, flip angle $=9^{\circ}, \mathrm{FOV}=256 \mathrm{~mm} \times 240 \mathrm{~mm}$, voxel size $=1$ $\mathrm{mm}^{3}, 176$ sagittal slices. Imaging parameters for the MP2-RAGE sequence were TR = $5000 \mathrm{~ms}, \mathrm{TE}=2.92 \mathrm{~ms}, \mathrm{TI}_{1} / \mathrm{TI}_{2}=700 \mathrm{~ms} / 2500 \mathrm{~ms}$, flip angle $1 /$ flip angle $_{2}=4^{\circ} / 5^{\circ}, \mathrm{FOV}=$ $256 \mathrm{~mm}$ x $240 \mathrm{~mm}$, voxel size $=1 \mathrm{~mm}^{3}, 176$ sagittal slices. All structural images were acquired using a 32-channel head coil, except for 2 participants where a 20 -channel coil was used. 


\title{
Data Analysis
}

\author{
Behavioural
}

Trials in which the participant failed to make a response, i.e., missed trials, were disregarded from analysis (6\% of trials). The overall trial count was then adjusted to include only trials on which a response was made. Accuracy was calculated as the number of correct responses divided by the adjusted trial count, for each participant, for each condition. Reaction times (in milliseconds) were also calculated for correct response trials, for each participant, for each condition. Behavioural data (voice-identity recognition performance: accuracy and reaction time) were analysed in Statistica (TIBCO Software) using a 2 x 2 repeated measures analysis of variance (ANOVA), with 'learning' (voice-face, voice-occupation) and 'noise-level' (high-noise, low-noise) as repeated factors. Effects were considered significant if present at $p<0.05$. Effect sizes were calculated using partial eta square $\eta_{p}^{2}$ (Cohen, 1969; Richardson, 2011).

\section{Functional MRI}

Functional MRI data were analysed with the statistical parametric mapping software package (SPM12, Wellcome Trust Centre for Neuroimaging, UCL, UK, (www.fil.ion.ucl.ac.uk/spm). We used standard spatial pre-processing procedures: images were realigned and unwarped, normalised to Montreal Neurological Institute (MNI) standard stereotactic space using the structural image of each participant, written to the original resolution $3 \times 3 \times 3 \mathrm{~mm}$, and smoothed with an isotropic Gaussian filter of $8 \mathrm{~mm}$ at FWHM. Geometric distortion due to susceptibility gradients were corrected by an interpolation procedure based on the B0 field-map. Statistical parametric maps were generated by modelling the evoked hemodynamic response for the different conditions as boxcars convolved with a synthetic hemodynamic response function in the context of the general linear model (Friston et al., 2007). All contrasts of interest were computed at the single-subject level and then taken to a group-level random-effects analysis which estimated the second-level $t$-statistic at each voxel.

\section{Regions of Interest}

Visual regions of interest (ROI) for the functional response and connectivity analyses were the FFA (Kanwisher, McDermott and Chun, 1997) and the pSTS-mFA (Pitcher et al., 2011). We localised the FFA with the contrast 'faces > objects'. The FFA was localised at the group level in the right hemisphere - maxima at $\mathrm{x}=45, \mathrm{y}=-40, \mathrm{z}=-19$ ( $T$-value $=$ 3.54). For the FFA ROI, the localiser was thresholded with a cluster size of 25 voxels. This cluster size is similar to previous reports for the FFA using this design and contrast (von Kriegstein et al., 2008; Borowiak, Maguinness and von Kriegstein, 2019). We did not 
observe responses in an analogous region in the left hemisphere at a threshold of $<0.01$ uncorrected. This right hemisphere dominance is in line with previous observations (Kanwisher, McDermott and Chun, 1997; von Kriegstein et al., 2008). The pSTS-mFA was localised using the contrast 'dynamic faces > dynamic objects' (Fox, Iaria and Barton, 2009). This contrast revealed a facial motion sensitive cluster in the right pSTS, with the maximum at the group level at $\mathrm{x}=54, \mathrm{y}=-34, \mathrm{z}=2(T$-value $=4.55)$ and an analogous cluster in the left hemisphere with the maximum at $\mathrm{x}=-51, \mathrm{y}=-46, \mathrm{z}=11(T$-value $=3.89)$. Both regions were thresholded to have a comparable cluster size of 29 voxels. All 3 visual ROIs were also localised using data from a sub-sample of those 16 participants that showed a behavioural face-benefit to facilitate sub-group analyses. Identical contrasts, as described above, were used to identify these ROIs in this group ( $\mathrm{N}=15$; one participant did not complete the localiser, see Participants section) (Supporting Information, Table 1).

The auditory ROIs for the functional connectivity analysis were the voice-sensitive regions in the right middle and anterior STG/S (Belin and Zatorre, 2003; von Kriegstein et al., 2003; von Kriegstein and Giraud, 2004; Schall et al., 2014). We chose these regions as they have been shown to share structural (Blank, Anwander and von Kriegstein, 2011) and functional (von Kriegstein et al., 2005; von Kriegstein and Giraud, 2006; von Kriegstein, Kleinschmidt and Giraud, 2006; Schall and von Kriegstein, 2014) connections with the FFA. We defined the mid and anterior STG/S using spheres (8mm radius) positioned around previously published co-ordinates in the right hemisphere for these regions (Blank, Anwander and von Kriegstein, 2011): mid STG/S: $x=63, y=-7, z=-14$; anterior STG/S: $x=$ $57, y=8, z=-11$. All ROI masks were created using SPM12.

\section{Contrasts of Interest}

We defined two different contrasts of interest to test whether BOLD responses increased in the visual ROIs during voice-identity recognition for voice-face learned speakers (in contrast to voice-occupation learned speakers) in high, compared to low, noise listening conditions. Firstly, we calculated contrast maps ( $t$-statistics), examining our central hypothesis, for the interaction term: ((voice-face/high-noise > voiceoccupation/high-noise) > (voice-face/low-noise > voice-occupation/low-noise)). Secondly, we performed post-hoc $t$-tests by investigating the effect of learning (voice-face vs. voiceoccupation) on responses in face-sensitive regions of the FFA and pSTS-mFA separately for both high- and low-noise listening conditions. Here, we calculated contrast maps $(t$ statistics) for the two simple main effects of learning for: high-noise listening conditions (voice-face/high-noise > voice-occupation/high-noise) and low-noise listening conditions (voice-face/low-noise $>$ voice-occupation/low-noise). Responses in each ROI were considered to be significant if they were present at $p<0.05$ family wise error (FWE) corrected for the ROI, Holm-Bonferroni (Holm, 1979) corrected for the number of ROIs (N =3). The Holm-Bonferroni method handles multiple comparisons via a sequential 
hypothesis rejection approach, and it is less susceptible to Type II error (i.e., missing true effects) compared to the standard single-step Bonferroni correction (Nichols and Hayasaka, 2003).

\section{Correlational Analyses}

For testing whether the magnitude of responses in face-sensitive regions during the voice-identity recognition task correlated with behavioural face-benefit scores across participants we performed the following steps. Firstly, we calculated the behavioural facebenefit score (von Kriegstein et al., 2008): \% correct voice-identity recognition for voiceface learning minus \% correct voice-identity recognition for voice-occupation learning. This score was calculated separately for high- and low-noise listening conditions, for each participant. We included the face-benefit score for high- and low-noise as a co-variate of interest in SPM12 for the second-level analysis of the simple main effects of learning for high- (voice-face/high-noise > voice-occupation/high-noise) and low- (voice-face/lownoise > voice-occupation/low-noise) noise listening conditions, respectively.

Secondly, as we noted in our previous behavioural study that the face-benefit increased in higher noise-levels (i.e., decreasing SNRs) (Maguinness, Schall and von Kriegstein, 2021), we calculated an additional score which reflected how well the behavioural face-benefit was maintained in the high-, relative to the low-, noise listening conditions (face-benefit high-noise - face-benefit low-noise). We refer to this score as "facebenefit maintenance". This score was calculated for each participant and was included in SPM12 as a co-variate of interest at the second-level analysis for the interaction contrast ((voice-face/high-noise > voice-occupation/high-noise) - (voice-face/low-noise > voiceoccupation/low-noise)).

The significance of the correlational analyses was assessed using SPM12 and considered to be significant if present at $p<0.05$ FWE corrected for the ROI, HolmBonferroni-corrected for the number of ROIs $(\mathrm{N}=3)$.

\section{Psychophysiological Interactions Analysis (PPI)}

To test whether there is functional connectivity (Friston, 1994), between visual face- and voice-sensitive regions during voice-identity recognition in high-noise, we conducted PPI analyses (Friston et al., 1997; O'Reilly et al., 2012). PPI analyses identify temporal correlations between responses in specific brain regions (i.e., seed regions) and responses in other brain regions (i.e., target regions) which are modulated by a psychological factor (i.e., experimental task). The seed regions for the PPI analysis were the visual face-sensitive regions (as defined by the visual face area localiser) which demonstrated selective enhanced responses for face-learned speakers in high-noise listening conditions ((voice-face/high-noise > voice-occupation/high-noise) > (voice- 
face/low-noise > voice-occupation/low-noise)). Target regions were the voice-sensitive mid and anterior STG/S.

We conducted the PPI analyses following standard procedures (Friston et al., 1997). We extracted the first Eigenvariate in the visual face-sensitive seed region and used the voice-sensitive regions in the mid and anterior STG/S regions as target regions. The analysis included the psychological variable for the simple main effects of learning for the high-noise listening condition (voice-face/high-noise > voice-occupation/high-noise). We modelled the first Eigenvariate, the psychological variable, and the psychophysiological interaction term as regressors at the single-subject level. The psychophysiological interaction term was created using routine procedures implemented in SPM12. Population-level inferences about BOLD signal changes were based on a random-effects model that estimated the second-level statistic at each voxel using a one-sample $t$-test. The face-benefit score for each participant, for the high-noise condition, was included as a co-variate of interest in the second-level analysis. Results were considered significant if they were present at $p<0.05$ FWE corrected for the target ROI, Holm-Bonferroni corrected for the number of target ROIs $(\mathrm{N}=2)$.

\section{Results}

Behavioural Results: Auditory-only Voice-identity Recognition

\section{Accuracy}

The $2 \times 2$ repeated measures analysis of variance (ANOVA), with 'learning' (voiceface or voice-occupation) and 'noise-level' (high-noise or low-noise) as repeated factors on the accuracy scores (Table 1) revealed a main effect of 'learning' $[F(1,20)=5.91, p=0.02$, $\left.\eta_{p}{ }^{2}=0.23\right]$. As expected, this main effect was based on higher accuracy for recognising the identity of speakers who had been previously learned through voice-face $(M=84.9 \%)$, rather than voice-occupation $(M=78.6 \%)$, learning. We refer to such an improvement in performance as the 'face-benefit' (von Kriegstein et al., 2008); 16 of the 21 participants showed this average effect across noise levels (Figure 5). A main effect of 'noise-level' was also observed $\left[\mathrm{F}(1,20)=37.75, p=<0.001, \eta_{\mathrm{p}}{ }^{2}=0.65\right]$, with lower recognition accuracy in high-noise $(M=77.9 \%)$, compared to low-noise $(M=85.6 \%)$, listening conditions. Contrary to our expectations, there was no significant interaction between 'learning' and 'noise-level' $\left[\mathrm{F}(1,20)=2.47, p=0.13, \mathrm{n}_{\mathrm{p}}{ }^{2}=0.10\right]$, suggesting that the difference in the face-benefit across noise conditions was not significant. The face-benefit in the high-noise condition was $5.4 \%$, and in the low-noise condition was 7.3\%. The face-benefit for high- and low-noise conditions was strongly positively correlated within participants (Pearson's $r=0.909, p=$ 
$0.000, \mathrm{~N}=21$; Pearson's $r=0.847, p=0.000, \mathrm{~N}=16$ positive face-benefit participants only; Figure 5).

Table 1. Behavioural accuracy and reaction times for auditory-only voice-identity recognition performance (with standard deviations) for voice-face and voice-occupation learned speakers, in high- and low-noise listening conditions.

\begin{tabular}{l|l|l}
\hline & high-noise & low-noise \\
\hline $\begin{array}{l}\text { voice-face } \\
\text { \% Correct }\end{array}$ & $80.6(7.5)$ & $89.3(8.6)$ \\
Reaction Time (ms) & $612.1(54.7)$ & $604.9(55.6)$ \\
\hline $\begin{array}{l}\text { voice-occupation } \\
\text { \% Correct }\end{array}$ & $75.1(11.1)$ & $82.0(9.6)$ \\
Reaction Time (ms) & $625.1(60.5)$ & $622.5(67.4)$ \\
\hline face-benefit & & \\
\% Correct & $5.4(12.9)$ & $7.3(11.6)$ \\
Reaction Time (ms) & $-12.9(32.6)$ & $-17.6(40.7)$ \\
\hline
\end{tabular}

ms = milliseconds; ${ }^{*}$ negative values indicate comparatively faster responses

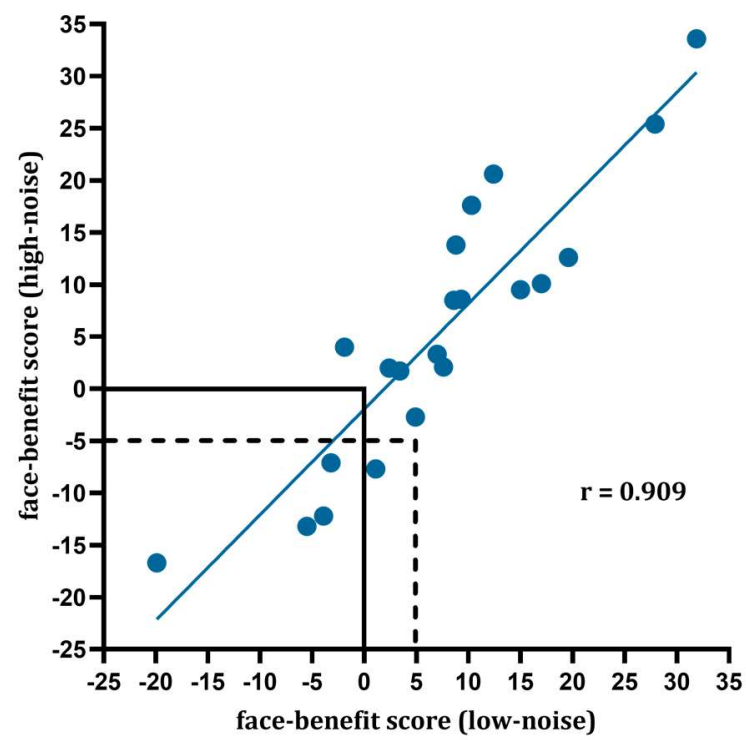

Figure 5. Plot showing the behavioural face-benefit score for each participant across high- (y axis) and low-noise ( $x$ axis) listening conditions. There was a significant positive correlation $(\mathrm{r}=$ $0.909, p=0.000$ ) between participants' face-benefit scores across noise levels. The bold black intersecting line denotes no face-benefit in either noise level (i.e., accuracy is equal for voice-face and voice-occupation learned speakers). The dashed intersecting line denotes a division between those with a positive $(\mathrm{N}=16)$ and negative $(\mathrm{N}=5)$ face-benefit score averaged over both noise levels. 


\section{Reaction Time}

A $2 \times 2$ repeated measures analysis of variance (ANOVA) was conducted on the time taken to recognise speaker identities across 'learning' (voice-face or voice-occupation) and 'noise-level' (high-noise or low-noise) conditions (Table 1). A main effect of 'learning' was found $\left[\mathrm{F}(1,20)=4.36, p=0.05, \eta_{\mathrm{p}}{ }^{2}=0.18\right]$. Participants were faster to recognise the identities of speakers who they had been previously learned through voice-face $(M=608$ $\mathrm{ms})$, compared to voice-occupation $(M=624 \mathrm{~ms})$, learning. This indicates that there was no speed accuracy trade-off for the main effect of learning. The main effect of 'noise-level' $\left[\mathrm{F}(1,20)=1.73, p=0.20, \eta_{\mathrm{p}}{ }^{2}=0.08\right]$ and the interaction between 'learning' and 'noise-level' $\left[\mathrm{F}(1,20)=0.49, p=0.49, \mathrm{n}_{\mathrm{p}}{ }^{2}=0.02\right]$ were not significant.

\section{Functional MRI Results}

\section{Increased responses in the right $p S T S-m F A$ during the recognition of face-learned speakers in high-noise}

To address our main hypothesis, we examined whether the recognition of facelearned speakers in higher versus lower noise was associated with increased responses in the FFA and/or the pSTS-mFA. To do this, we used the interaction contrast ((voiceface/high-noise $>$ voice-occupation/high-noise) $>$ (voice-face/low-noise $>$ voiceoccupation/low-noise)). For this contrast, we observed increased responses in the right pSTS-mFA ( $\mathrm{x}=51, \mathrm{y}=-37, \mathrm{z}=8, \mathrm{p}=0.012$, FWE corrected for ROI, Holm-Bonferroni corrected for number of ROIs; Figure 6A-B). There were also increased responses in the left pSTS-mFA, although this did not survive Holm-Bonferroni-correction $(\mathrm{x}=-54, \mathrm{y}=-43, \mathrm{z}=8$, $p=0.027$, FWE corrected for the ROI; Supporting Information, Figure 2). Contrary to our expectations, no increased responses were observed in the FFA for the interaction contrast even at a lenient threshold ( $p<0.05$, uncorrected). To confirm the directionality of the interaction we examined responses in the visual ROIs for face-learned speakers separately for high- and low-noise listening conditions. The tests of simple main effects of learning confirmed increased responses in the right pSTS-mFA ( $\mathrm{x}=51, \mathrm{y}=-34, \mathrm{z}=1, p=0.028$, FWE corrected for the ROI) for the contrast (voice-face/high-noise > voice-occupation/highnoise). In contrast, there was no evidence for increased responses in the left or right pSTSmFA or FFA for the contrast (voice-face/low-noise > voice-occupation/low-noise), even at lenient thresholds $(p<0.05$, uncorrected).

We noted, in line with previous findings (von Kriegstein et al., 2008; Maguinness, Schall and von Kriegstein, 2021), that not all participants had a behavioural face-benefit (Figure 5). To further examine the functional relevance of responses in the visual ROIs for the face-benefit on speaker recognition, we separately examined responses for the 16 participants who showed this behavioural enhancement. The responses in the visual ROIs 
(Supporting Information, Table 1, ROIs for $\mathrm{N}=15$ ) stayed qualitatively the same: there was a noise-level $x$ learning interaction in the right pSTS-mFA ( $\mathrm{x}=54, \mathrm{y}=-28, \mathrm{z}=-1, p=0.030$, FWE corrected for ROI), which did, however, not survive Holm-Bonferroni correction. The left pSTS-mFA was not significant ( $\mathrm{x}=-54, \mathrm{y}=-43, \mathrm{z}=8, p=0.097$, FWE corrected for ROI). Tests of simple main effects of learning showed significantly increased responses in the right pSTS-mFA ( $\mathrm{x}=51, \mathrm{y}=-31, \mathrm{z}=-1, p=0.004$, FWE corrected for the ROI, HolmBonferroni corrected for number of ROIs), for the contrast (voice-face/high-noise $>$ voiceoccupation/high-noise). Responses for this contrast in the left pSTS-mFA ( $\mathrm{x}=-51, \mathrm{y}=-43, \mathrm{z}$ $=8, p=0.042$, FWE corrected for the ROI) did not survive Holm-Bonferroni correction. No increased responses were observed in the left or right pSTS-mFA or FFA for the contrast "voice-face/low-noise > voice-occupation/low-noise", even at lenient thresholds $(p<0.05$, uncorrected). Taken together, these findings suggest that motion-sensitive regions of the face-network, particularly in the right hemisphere, may be engaged for voice-identity recognition in high-noise listening conditions.

The face-benefit is positively correlated with increased functional responses in the FFA and the right pSTS-mFA in low-noise listening conditions

Previous studies have demonstrated responses in the FFA during the recognition of speakers known by face (von Kriegstein et al., 2005; von Kriegstein and Giraud, 2006; von Kriegstein, Kleinschmidt and Giraud, 2006; Blank, Anwander and von Kriegstein, 2011; Schall et al., 2013). Pertinently, these responses have been shown to be behaviourally relevant for supporting voice-identity recognition: They correlated positively with the facebenefit score (von Kriegstein et al., 2008) in typically developed individuals, but not in developmental prosopagnosics (individuals with a severe deficit in face-identity processing; McConachie, 1976) who do not have a face-benefit (von Kriegstein, Kleinschmidt and Giraud, 2006; von Kriegstein et al., 2008). The categorical results from the previous section suggest that the pSTS-mFA may be involved in voice-identity recognition in more noisy listening conditions, although the behavioural relevance of these responses remains unclear. Therefore, we examined a possible correlation between the behavioural face-benefit and responses in the visual ROIs for both the high- (voice-face/high-noise > voice-occupation/ high-noise) and low- (voice-face/low-noise > voice-occupation/lownoise) noise listening conditions. Contrary to our expectations, in high-noise listening conditions, the face-benefit (calculated for the high-noise condition) did not correlate with responses in any visual ROIs for the contrast "voice-face/high-noise > voiceoccupation/high-noise". In low-noise listening conditions, we found a significant positive correlation between the face-benefit (calculated for the low-noise condition) and responses in the FFA ( $\mathrm{x}=48, \mathrm{y}=-40, \mathrm{z}=-16$; $p=0.000$; FWE corrected for ROI, Holm-Bonferroni corrected for number of ROIs). There was also a positive correlation between the behavioural face-benefit and responses in the right pSTS-mFA in the low-noise condition ( $\mathrm{x}$ $=57, \mathrm{y}=-31, \mathrm{z}=5, p=0.021$; FWE corrected for the ROI, Holm-Bonferroni corrected for 
number of ROIs; Figure 6C-D). For the analyses with the 16 participants with a face-benefit the low-noise listening condition results for the FFA remained ( $p=0.006$; FWE corrected for ROI, Holm-Bonferroni corrected for number of ROIs). However, the pSTS-mFA (left or right) did not show a significant correlation in this sub-group ( $p>0.05$, uncorrected).

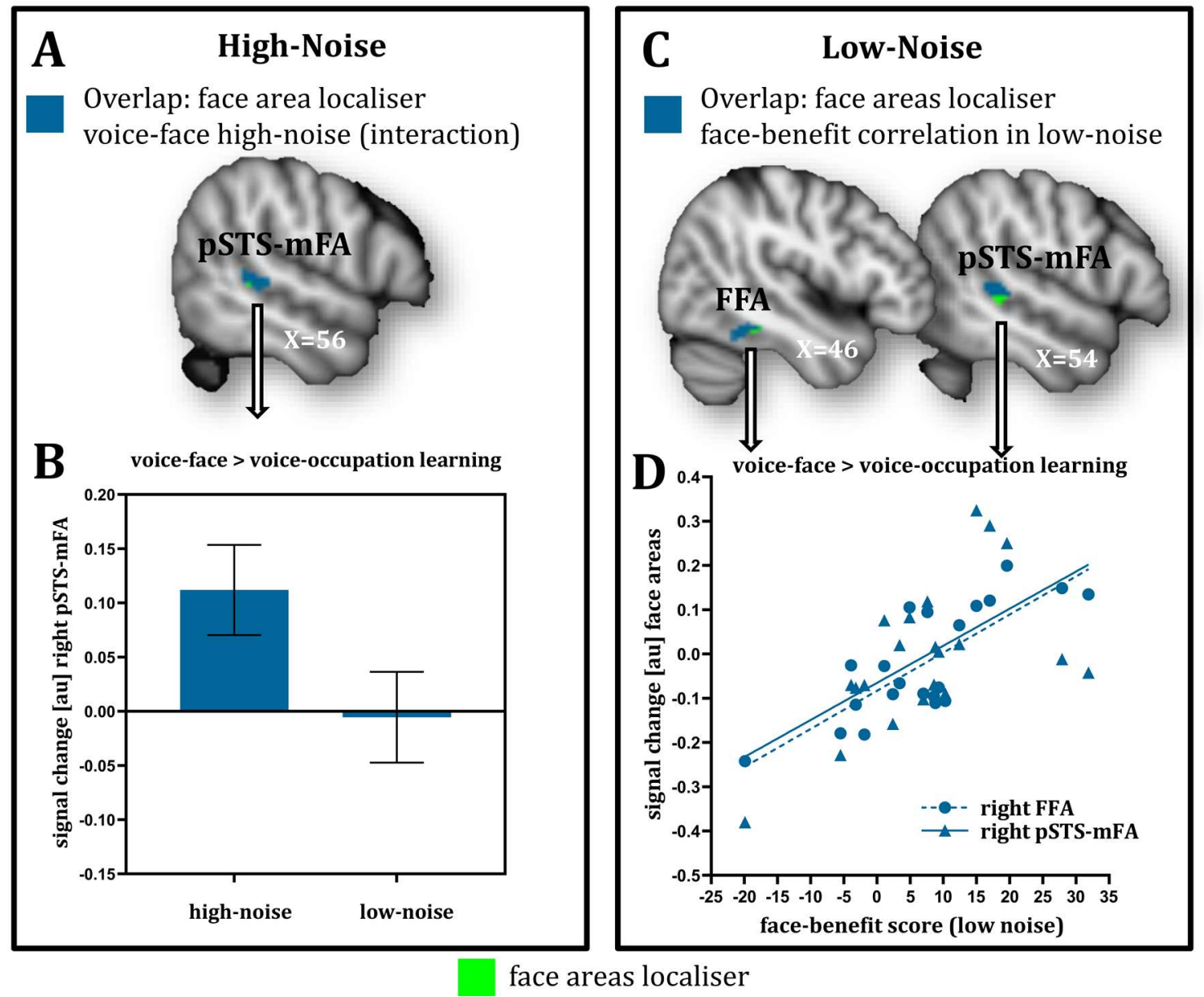

Figure 6. fMRI results. Overview of responses in visual face-areas (FFA, pSTS-mFA) during voiceidentity recognition for face-learned speakers in different levels of auditory noise. (A) and (B) HighNoise. (A) Overlay of the interaction contrast ((voice-face high-noise > voice-occupation high-noise) $>$ (voice-face low-noise > voice-occupation low-noise)) and the functional face-area localiser contrast for the right pSTS-mFA (dynamic faces $>$ dynamic objects) (overlap between the contrasts is shown in blue). The maximum statistic for the interaction contrast was at $\mathrm{x}=51, \mathrm{y}=-37, \mathrm{z}=8, \mathrm{p}=0.012$, FWE corrected for ROI, Holm-Bonferroni corrected for number of ROIs. (B) Signal change estimate in the right pSTS-mFA for conditions that were included in the interaction contrast ((voice-face highnoise > voice-occupation high-noise) $>$ (voice-face low-noise > voice-occupation low-noise)) during auditory-only voice-identity recognition. Plot shows first eigenvariate extracted from the maximum statistic for the interaction contrast shown in A. (C) and (D) Low-noise. (C) Overlay of the correlation between the face-benefit score for each participant and the contrast (voice-face low-noise $>$ voice-occupation low-noise) and the functional localiser contrasts for the right FFA (faces > objects) and right pSTS-mFA (dynamic faces > dynamic objects) (overlap between the contrasts is 
shown in blue). (D) Plot of the correlation between the face-benefit score and responses in right FFA and right pSTS-mFA for the contrast (voice-face > voice-occupation) in low-noise listening conditions. The signal change estimate (first eigenvariate) was extracted from the peak co-ordinates for the right FFA ( $\mathrm{x}=48, \mathrm{y}=-40, \mathrm{z}=-16, p=0.000$, FWE corrected for ROI, Holm-Bonferroni corrected for number of ROIs) and the right pSTS-mFA ( $\mathrm{x}=57, \mathrm{y}=-31, \mathrm{z}=5, p=0.021$, FWE corrected for ROI, Holm-Bonferroni corrected for number of ROIs) for the contrast (voice-face low-noise $>$ voiceoccupation) in low-noise, with the face-benefit score (low-noise) as a co-variate of interest. The plot is shown for display purposes only. Statistics were computed in SPM12 (see Methods). In $\mathbf{A}$ and $\mathbf{C}$ the overlapping responses (blue colours) between the voice-identity recognition contrasts and the faceareas are presented for display purposes at $p=0.05$ whole brain uncorrected, masked by the ROI. Non-overlapping responses in visual face-areas are shown in green. All responses are overlaid on a mean structural MNI152 T1 weighted image (sagittal view, right hemisphere) and visualised using MRIcron (www.nitrc.org/projects/mricron). All co-ordinates are reported in MNI-space.

Are responses in the right $p S T S-m F A$ associated with the ability to maintain the facebenefit in high-noise listening conditions?

It was surprising that in high-noise conditions there were no positive correlations between BOLD responses in the pSTS-mFA and the face-benefit score. This could mean that the recruitment of pSTS-mFA during high-noise reflects an attempt to compensate, but ultimately fails to be of behavioural relevance for supporting voice-identity recognition. Previously, we observed that for participants who have a face-benefit (76\% of participants - Maguinness, Schall and von Kriegstein, 2021), there is a linear increase in the face-benefit with increasing auditory noise. Contrary to this, we did not observe a larger face-benefit in the high-noise condition of the present study (see Table 1). However, we noted variability in participants behavioural performance - not all participants maintained the same degree of face-benefit across noise levels. For some this benefit dropped substantially in the high-, compared to low-noise, condition. While for others the face-benefit was equatable or even greater in high-noise (Figure 5). An alternative view may therefore be that the pSTS-mFA may be behaviourally relevant for maintaining the face-benefit in noise. To that end, we conducted an exploratory analysis which examined the correlation between the "facebenefit maintenance" score (face-benefit high-noise minus face-benefit low-noise) and the interaction contrast ((voice-face/high-noise > voice-occupation/high-noise) > (voiceface/low-noise > voice-occupation/low-noise)) in the visual ROIs. The correlation was not statistically significant in the right pSTS-mFA $(\mathrm{x}=63, \mathrm{y}=-43, \mathrm{z}=8 ; p=0.069$, FWE corrected for the ROI) or the other two ROIs even at lenient thresholds ( $p<0.05$, uncorrected). When the analysis included only the 16 participants who had demonstrated a face-benefit on speaker recognition there was a positive correlation between the face-benefit maintenance score and responses in the right pSTS-mFA ( $\mathrm{x}=48, \mathrm{y}=-34, \mathrm{z}=-4 ; p=0.024$, FWE corrected for the ROI), although this did not survive Holm-Bonferroni correction for the 3 ROIs (Figure 7). Neither the left pSTS-mFA nor the FFA correlated with this measure in this sub-group ( $p$ $>0.05$, uncorrected). This finding suggests a potential behaviourally relevant role for the right pSTS-mFA in preserving the beneficial effect of face experience on voice-identity 
recognition in high-levels of auditory noise, for participants who benefit from audio-visual voice-face learning. However, given that it did not survive Holm-Bonferroni correction and was an exploratory analysis it must be taken with caution.

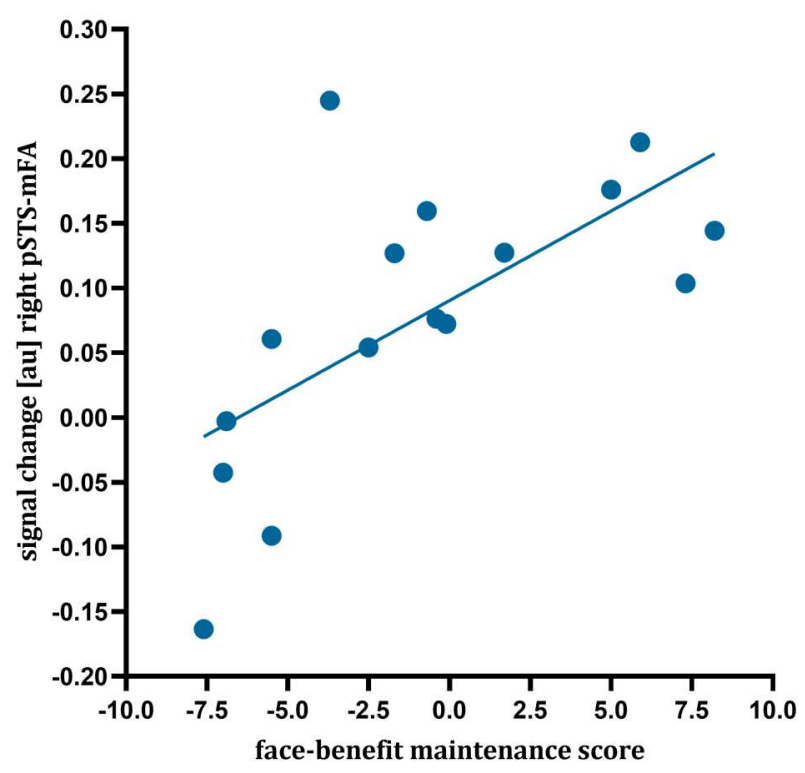

Figure 7. Plot showing the correlation between the face-benefit maintenance score $(\mathrm{N}=16$ facebenefit participants) and functional responses in the right pSTS-mFA (first eigenvariate extracted from peak co-ordinate $\mathrm{x}=48, \mathrm{y}=-34, \mathrm{z}=-4, p=0.024$, FWE corrected for ROI) for the interaction contrast ((voice-face high-noise > voice-occupation high-noise) $>$ (voice-face low-noise $>$ voiceoccupation low-noise)). The correlation was computed in SPM12 and the plot serves for display purposes, only. The correlation did not survive Holm-Bonferroni correction for three ROIs and therefore should be interpreted with caution.

Functional connectivity between $p S T S-m F A$ and voice-sensitive regions in the right $S T G / S$ is associated with the face-benefit in high-noise listening conditions

In previous work it was shown that the FFA is functionally coupled with voicesensitive regions in the anterior and mid STG/S during voice-identity recognition of facelearned speakers (von Kriegstein et al., 2005; von Kriegstein and Giraud, 2006; von Kriegstein, Kleinschmidt and Giraud, 2006; Schall and von Kriegstein, 2014). Based on these findings we expected that the right pSTS-mFA, associated with the recognition of facelearned speakers in high-noise (Figure 6A, B), would also share functional connections with voice-sensitive regions in the STG/S. To test whether the pSTS-mFA is functionally connected to anterior and mid STG/S voice-sensitive regions, we conducted PPI analyses, including the psychological variable (voice-face high-noise > voice-occupation high-noise). We defined the right pSTS-mFA as the seed region and the voice-sensitive mid STG/S and anterior STG/S as target regions. There were no significant results in any of the target ROIs, 
even at a lenient threshold of $p<0.05$ uncorrected, for analyses including $\mathrm{N}=21$, or $\mathrm{N}=16$ face-benefit participants.

Next, we explored whether there might be a correlation between functional connectivity of face- and voice-sensitive regions in the STG/S during voice-identity recognition in high-noise listening conditions and the amount of the face-benefit across participants. The analysis included the psychological variable (voice-face high-noise > voice-occupation high-noise) and the behavioural covariate (face-benefit high-noise) for all participants $(\mathrm{N}=21)$. The correlation for all $\mathrm{N}=21$ between the face-benefit and increased functional connectivity between the right pSTS-mFA (seed region) and either voicesensitive regions in the mid or anterior STG/S was not statistically significant (mid STG/S at $\mathrm{x}=63, \mathrm{y}=-4, \mathrm{x}=-10, p=0.064$, FWE corrected for the ROI; anterior STG/S at $\mathrm{x}=60, \mathrm{y}=$ $2, \mathrm{x}=-7, p=0.098$, FWE corrected for the ROI). Interestingly, the correlation was only significant for the 16 participants who displayed a face-benefit on voice-identity recognition: This was the case for both the connectivity between the right pSTS-mFA and the mid voice-sensitive STG/S $(\mathrm{x}=63, \mathrm{y}=-1, \mathrm{z}=-10 ; p=0.008$, FWE corrected for ROI, Holm-Bonferroni corrected for number of ROIs) and the anterior voice-sensitive STG/S ( $\mathrm{x}$ $=60, \mathrm{y}=2, \mathrm{z}=-7 ; p=0.024$, FWE corrected for ROI, Holm-Bonferroni corrected for number of ROIs) (Figure 8; Table 2).

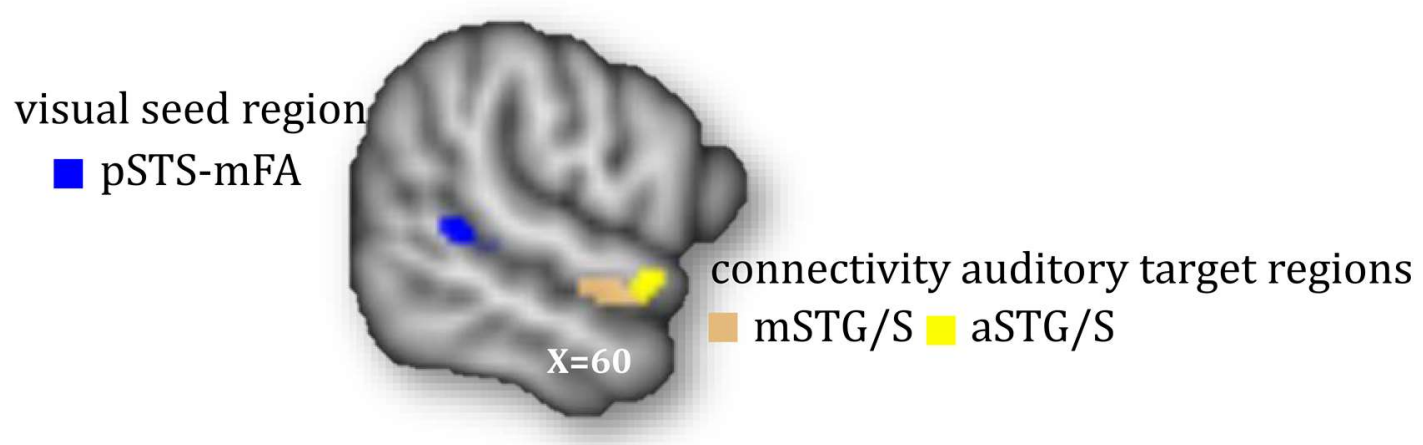

Figure 8. Functional connectivity between face- and voice-sensitive regions during voiceidentity recognition for face-, in comparison to occupation-, learned speakers in high-noise. In high-noise listening conditions, in participants who benefitted from voice-face learning $(\mathrm{N}=$ 16), the face-benefit correlated positively with increased connectivity between the right pSTS-mFA (blue; dynamic faces > dynamic objects) and auditory voice-sensitive regions in the mid and anterior STS/G (beige/yellow). For display purposes, results for auditory target regions are shown at 0.05 whole brain uncorrected, masked by the $8 \mathrm{~mm}$ spheres centered on previously published coordinates for the mid and anterior STG/S (Blank, Anwander and von Kriegstein, 2011). 
Table 2. Peak co-ordinates for voice-sensitive regions which showed increased functional connectivity to the right pSTS-mFA during the recognition of voice-face learned, in contrast to voiceoccupation learned, speakers in high-noise listening conditions.

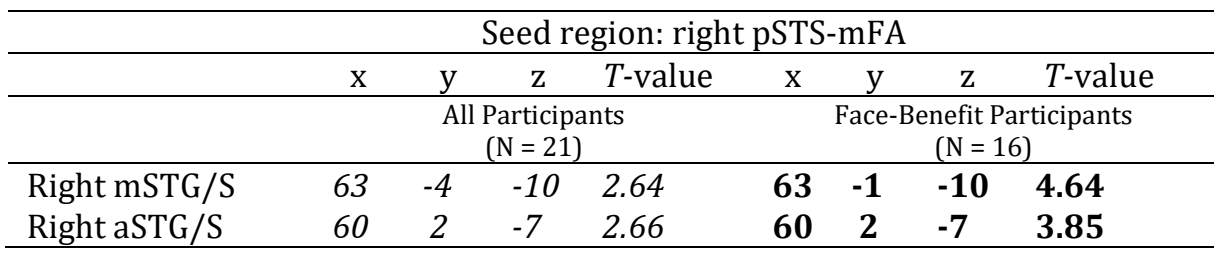

Co-ordinates in bold are significant for the face-benefit participants ( $\mathrm{N}=16$, right $\mathrm{mSTG} / \mathrm{S} p=0.008$, aSTG/S $p=0.024$, FWE corrected for ROI, Holm-Bonferroni corrected for number of ROIs), coordinates in italics are non-significant $(\mathrm{N}=21$, all participants) and are included for comparison purposes only.

\section{Discussion}

We investigated whether the visual mechanisms used during the recognition of auditory-only communication signals are adaptive to different levels of noise in the auditory signal. Our study had two key findings. Firstly, and centrally, in high-noise listening conditions we observed that the recognition of face-learned speakers recruited the motionsensitive right pSTS-mFA. Unexpectedly, responses in this region did not correlate with listeners' behavioural face-benefit scores. Only the functional connectivity between the right pSTS-mFA and voice-sensitive regions correlated with the behavioural face-benefit in the high-noise listening condition, in the 16 participants with a face-benefit. Conversely, in low-noise, there was no categorical response to face-learned, in contrast to occupation learned, speakers in any visual face-sensitive ROI. However, in this noise level, the behavioural face-benefit was robustly correlated with increased functional responses in the region sensitive to structural facial-identity cues i.e., the FFA and - to some extent - with the right $\mathrm{pSTS}-\mathrm{mFA}$. The findings suggest that partially distinct visual mechanisms support the face-benefit in different levels of auditory noise.

\section{Visual Responses during Voice-identity Recognition in Noise: Support for an} Audio-visual Model of Human Auditory Communication

The visual pSTS-mFA has been implicated in the processing of dynamic facial cues, including those dynamic cues which support identity processing (O'Toole, Roark and Abdi, 2002; Girges, Spencer and O'Brien, 2015; Girges, O’Brien and Spencer, 2016). Our findings suggest that voice-identity recognition in high-noise, when listeners arguably attend to more dynamic aspects of the voice for recognition, may stimulate the engagement of stored dynamic, rather than static, identity cues encoded during audio-visual voice-face learning. Such a finding corroborates previous observations that voice-identity recognition is 
facilitated by dynamic identity cues available in the auditory and visual streams. For example, Schweinberger and Robertson (2017) noted that familiar voices were more readily recognised when accompanied with their time synchronised face (i.e., video), while recognition was impaired when the corresponding moving face was presented with a temporal asynchrony. The present findings imply that even in the absence of the concurrent face input, the recruitment of a brain region implicated in dynamic face processing may support voice-identity processing, particularly when static auditory cues are degraded.

Conversely, in low-noise listening conditions, when recognition may rely on more available static vocal properties (Figure 2), we noted a robust relationship between the facebenefit and responses in the FFA. Responses in this facial form-sensitive region correlated with individuals' recognition scores for face, in comparison to non-face, learned speakers. While we found a similar correlation with responses in the right pSTS-mFA, this correlation did not survive for individuals who displayed a positive face-benefit score. Together, these findings of responses in different face-sensitive regions, modulated by noise in the auditory signal, highlight the remarkably adaptive nature of cross-modal responses observed under unisensory listening conditions. They support central assumptions of the audio-visual model of human auditory communication (von Kriegstein, 2012) by demonstrating that: 1) the visual regions engaged during auditory-only processing may be linked to the common source, task relevant, identity information available in both modalities (i.e., dynamic or static cues); 2) persistent responses in visual regions during noisy listening conditions serve to enhance the processing of voices, rather than being an epiphenomenon of successful speaker-identity recognition (von Kriegstein, Kleinschmidt and Giraud, 2006; Schall et al., 2013).

\section{The Face-benefit across Noise-levels}

Based on previous behavioural findings (Maguinness, Schall and von Kriegstein, 2021) we had expected that the face-benefit would be greatest in the high-noise condition. Surprisingly, this was not the case. There are two likely reasons for the lack of difference across noise levels. One may relate to the number of SNRs tested. Our previous observation of a linear increase in the face-benefit with increasing noise (Maguinness, Schall and von Kriegstein, 2021) tested a greater number of SNRs. Potentially, the linear effect may be more apparent with this larger range. However, the low-noise condition in the present study was associated with a quantitatively (but not significantly) higher face-benefit than the highnoise condition. Another possibility is therefore that the face-benefit increases linearly with reduced SNRs (Maguinness, Schall and von Kriegstein, 2021), but starts to break down at a certain point where the SNR is so low that static, and potentially also dynamic cues, cannot be tracked in an optimal manner. Although our previous study showed the linear effect up to SNR of $-8 \mathrm{~dB}$, in the present study we were faced with more challenging listening conditions, considering the additional MRI-machine noise. 


\section{Relevance of the pSTS-mFA for the Face-benefit}

Unlike the FFA, responses in the right pSTS-mFA, during either low- or high-noise listening conditions, did not correlate directly with the (positive) face-benefit score. These findings might question the behavioural relevance of responses in the pSTS-mFA. While this lack of correlation may relate to the saliency of the dynamic cues available in the SNRs tested, it is additionally possible that it may also relate to the difference in the time courses for the acquisition of structural form and dynamic identity cues. For example, although dynamic facial identity cues can be learned, it has been suggested that they become more robust with repeated exposure and the degree of idiosyncrasy (O'Toole, Roark and Abdi, 2002; Roark et al., 2003; Lander and Chuang, 2005; Butcher and Lander, 2017). Thus, during our audiovisual training the acquired dynamic identity signature may have been less robust, than its corresponding more rapidly acquired structural identity representation (Blank, Kiebel and von Kriegstein, 2015). This may possibly explain why the face-benefit correlated directly with FFA responses, but not with responses in the pSTS-mFA. Alternatively, responses in the pSTS-mFA may not be as stable at supporting identity processing compared to structural cues in the FFA. For example, while individuals with developmental prosopagnosia can use dynamic cues to recognise faces in laboratory settings (Steede, Tree and Hole, 2007; Longmore and Tree, 2013), they nevertheless fail to recognise faces in day-to-day interactions, highlighting that dynamic cues alone may not be sufficient to support typical identity processing (Maguinness and Newell, 2015). Notwithstanding these considerations, the connectivity results from the main fMRI experiment support behaviourally relevant cross-modal interactions between dynamic face and voice regions: as the face-benefit (in participants who benefitted for voice-face learning) in high-noise correlated with increased functional connectivity between the pSTS-mFA and the voicesensitive STG/S. We take this behaviourally relevant connectivity profile as first evidence that dynamic face cues and processing in the pSTS-mFA may support the auditory-only processing of face-learned speakers, particularly in degraded listening conditions. Interestingly, functional connections between the left face-sensitive pSTS and a speech intelligibility region in the left anterior STS have been shown to support speech recognition for face learned speakers (Schall and von Kriegstein, 2014). While we corroborate a similar AV network, we demonstrate that for voice-identity recognition it is likely associated with responses in the right pSTS. This connectivity profile is in line with the right hemisphere's dominant role in identity processing (Assal et al., 1976; De Renzi, 1986; Kanwisher, McDermott and Chun, 1997; Belin and Zatorre, 2003; von Kriegstein and Giraud, 2004; Barton, 2008; Rossion, 2014; Liu et al., 2015; Luzzi et al., 2018).

\section{An Audio-visual Voice-face Network along the STS for Voice-identity Processing}

Recently, Yovel and 0'Toole (2016) proposed that recognition of the "dynamic speaking person" was likely mediated solely by voice and face processing regions along the 
STS which are sensitive to temporal information and dismissed a potential role for interactions with the FFA. Importantly, while we documented evidence of a motionsensitive AV network we demonstrate that it is likely complementary, rather than fundamental, for supporting voice-identity recognition. In a similar vein to face-identity recognition, the network appears to be recruited as a complementary, potentially "backup", system for supporting voice-identity recognition when static cues are altered or unavailable. We propose that the AV voice-face network along the STS might systematically supplement the FFA mechanism, i.e., becoming increasingly more responsive, as static aspects of the auditory signal are degraded. This is suggested by our finding of behaviourally relevant responses in both the FFA and to a lesser degree in the right pSTS-mFA during voice-identity recognition in low-noise. Conversely, in high-noise the recognition of facelearned speakers engaged the pSTS-mFA only. Such a system is in line with the visual literature which has demonstrated that the perceptual system integrates both static and dynamic face-identity cues in a manner which is dependent on either cues perceived saliency (Knappmeyer, Thornton and Bülthoff, 2003; Dobs, Ma and Reddy, 2017). While Yovel and O'Toole (2016) and other AV models of person-identity processing are mostly tailored towards the brain mechanisms supporting audio-visual integration i.e., when both face and voice are concurrently presented (e.g., Young, Frühholz and Schweinberger, 2020), our findings nevertheless highlight the importance of considering how both dynamic and static AV identity cues might be integrated. Given the perceptual system's sensitivity to static and dynamic components in the AV person-identity signal, we deem it is unlikely that integration is governed solely by a common global mechanism in the STS.

\section{The pSTS as a Multimodal Region and Voice-sensitive Region}

The posterior STS has been associated with the multimodal representation and integration of faces and voices (Tsantani et al., 2019; Young, Frühholz and Schweinberger, 2020), compared to e.g., objects and sounds (Watson et al., 2014). Thus, the region could be conceived as an audio-visual person-identity representational hub, rather than a region which is sensitive to different aspects of identity including facial dynamics. Theoretically, it is possible that a multimodal pSTS may be additionally recruited to support the recognition of face-learned voices in noise. However, two points speak against this. Firstly, we functionally localised the motion-sensitive face-area in the pSTS with a specific localiser contrasting dynamic faces against dynamic objects (group $(\mathrm{N}=20)$ peak voxel location: $\mathrm{x}=$ $54, y=-34, z=2$ ) and localised the peak responses for face-learned speakers in high-noise at $\mathrm{x}=51, \mathrm{y}=-37, \mathrm{z}=8$ (group $(\mathrm{N}=21$ ) peak voxel location; noise-level $\mathrm{x}$ learning interaction). The peak response for the interaction was $>1 \mathrm{~cm}$ away from those reported for multimodal voice-face representations $(x=48, y=-49, z=11$; searchlight analysis for cross-modal generalisation of discriminants for pairs of identities (Tsantani et al., 2019)). Secondly, if the observed pSTS responses were an additional audio-visual integrative 
mechanism recruited to complement the FFA, we would expect FFA responses to be equally present for the high-noise conditions. This was not the case.

Additionally, the pSTS has been implicated in voice-identity processing, particularly for unfamiliar voices which require increased perceptual processing (von Kriegstein and Giraud, 2004; Schelinski, Borowiak and von Kriegstein, 2016). Thus, it could be argued that the observed enhanced pSTS responses in the current study may have been driven solely by increased voice-identity processing in more challenging listening conditions. However, if this were the case, we would expect an overall (i.e., regardless of learning condition) response increase in this region during voice-identity processing in high-, compared to low, noise listening conditions. This was not evident. In contrast, the pSTS-mFA responses were observed specifically for face-learned speakers in noise (i.e., interaction effect) and not as a main effect for processing voices in noisier listening conditions (see Supporting Information, Functional MRI Analysis).

Inter-individual Variability in the Face-benefit

We noted variability in how well participants maintained the face-benefit in high-, compared to, low-noise listening conditions. Based on an exploratory analysis, there were some indications that this variability may relate to responses in the right pSTS-mFA, such that higher face-benefit maintenance scores were correlated with increased functional responses within this region. However, it is important to note that this correlation analysis was exploratory and did not survive Holm-Bonferroni correction and should be interpreted with caution. This observation was restricted to the 16 individuals who benefitted from face-voice learning, i.e., $76 \%$ of the tested sample. Currently it is unclear why some individuals do not benefit from face-voice learning. Although findings from developmental prosopagnosia (McConachie, 1976), i.e., a severe deficit in face-identity processing, suggest that it may be related to face processing abilities (von Kriegstein, Kleinschmidt and Giraud, 2006; von Kriegstein et al., 2008; Maguinness and von Kriegstein, 2017). Other evidence of interactions (Bülthoff and Newell, 2015, 2017) and relationships between face- and voiceidentity recognition abilities in the neurotypical population (Jenkins et al., 2020), suggest that a common coding system may underpin this enhancement i.e., similar computations in different modalities. Interestingly, the proportion of the current sample with a face-benefit is in line with our previous observations. For example, von Kriegstein et al. (2008) observed a face-benefit for voice-identity recognition in 13 of the 17 participants tested. While in Maguinness et al. (2021) this face-benefit was present in 19 of 25 individuals.

Conclusion

In summary, we propose that during audio-visual learning a vocal identity becomes enriched with distinct visual features, pertaining to both static and dynamic aspects of facial 
identity. These stored visual cues are used in an adaptable manner, tailored to perceptual demands, to optimise subsequent auditory-only voice-identity recognition. In more optimal listening conditions, the FFA is recruited to enhance voice-identity recognition. In contrast, under more degraded listening conditions, the facial motion-sensitive pSTS-mFA is recruited, although this complementary mechanism may be potentially less beneficial for supporting voice-identity recognition than that of the FFA. Taken together, these findings corroborate and extend an audio-visual view of human auditory communication, providing evidence for the particularly adaptive nature of cross-modal responses and interactions observed under unisensory listening conditions.

\section{References}

Assal, G. et al. (1976) '[Voice discrimination in patients with cerebral cortical lesions]', Schweizer Archiv fur Neurologie, Neurochirurgie und Psychiatrie = Archives suisses de neurologie, neurochirurgie et de psychiatrie. 1976/01/01, 119(2), pp. 307-315. Available at: http://www.ncbi.nlm.nih.gov/pubmed/1006205.

Axelrod, V. and Yovel, G. (2015) 'Successful decoding of famous faces in the fusiform face area', PLoS ONE, 10(2), p. e0117126. doi: 10.1371/journal.pone.0117126.

Barton, J. J. S. (2008) 'Structure and function in acquired prosopagnosia: lessons from a series of 10 patients with brain damage.', Journal of Neuropsychology. British Psychological Society, 2(1), pp. 197-225. doi: 10.1348/174866407X214172.

Belin, P. and Zatorre, R. J. (2003) 'Adaptation to speaker's voice in right anterior temporal lobe', Neuroreport, 14(16), pp. 2105-2109. doi: 10.1097/01.wnr.0000091689.94870.85.

Bernstein, M. et al. (2018) 'An Integrated Neural Framework for Dynamic and Static Face Processing', Scientific Reports. Springer US, 8(1), pp. 2-11. doi: 10.1038/s41598018-25405-9.

Bernstein, M. and Yovel, G. (2015) 'Two neural pathways of face processing: A critical evaluation of current models', Neuroscience and Biobehavioral Reviews. Elsevier Ltd, 55, pp. 536-546. doi: 10.1016/j.neubiorev.2015.06.010.

Blank, H., Anwander, A. and von Kriegstein, K. (2011) 'Direct structural connections between voice- and face-recognition areas.', The Journal of Neuroscience, 31(36), pp. 12906-12915. doi: 10.1523/JNEUROSCI.2091-11.2011.

Blank, H., Kiebel, S. J. and von Kriegstein, K. (2015) 'How the human brain exchanges information across sensory modalities to recognize other people', Human Brain Mapping, (36), pp. 324-339.

Borowiak, K., Maguinness, C. and von Kriegstein, K. (2019) 'Dorsal-movement and ventralform regions are functionally connected during visual-speech recognition', Human 
Brain Mapping. doi: 10.1002/hbm.24852.

Bülthoff, I. and Newell, F. N. (2015) 'Distinctive voices enhance the visual recognition of unfamiliar faces', Cognition, 137, pp. 9-21. doi: 10.1016/j.cognition.2014.12.006.

Bülthoff, I. and Newell, F. N. (2017) 'Crossmodal priming of unfamiliar faces supports early interactions between voices and faces in person perception', Visual Cognition, 24, pp. 611-628. doi: 10.1080/13506285.2017.1290729.

Butcher, N. and Lander, K. (2017) 'Exploring the motion advantage: evaluating the contribution of familiarity and differences in facial motion', Quarterly Journal of Experimental Psychology. Taylor \& Francis, 70(5), pp. 919-929. doi: 10.1080/17470218.2016.1138974.

Cohen, J. (1969) Statistical Power Analysis for the Behavioural Sciences. New York: Academic Press.

Dellwo, V., Leemann, A. and Kolly, M.-J. (2015) 'Rhythmic variability between speakers: Articulatory, prosodic, and linguistic factors', The Journal of the Acoustical Society of America, 137(3), pp. 1513-1528. doi: 10.1121/1.4906837.

Dobs, K., Bülthoff, I. and Schultz, J. (2016) 'Identity information content depends on the type of facial movement', Scientific Reports, 6(May), pp. 1-9. doi: 10.1038/srep34301.

Dobs, K., Ma, W. J. and Reddy, L. (2017) 'Near-optimal integration of facial form and motion', Scientific Reports. Springer US, 7(1), pp. 1-9. doi: 10.1038/s41598-017-10885-y.

Eger, E., Schyns, P. G. and Kleinschmidt, A. (2004) 'Scale invariant adaptation in fusiform face-responsive regions', NeuroImage, 22, pp. 232-242. doi: 10.1016/j.neuroimage.2003.12.028.

Erber, N. P. (1969) 'Interaction of Audition and Vision in the Recognition of Oral Speech Stimuli', Journal of Speech, Language, and Hearing Research, 12(2), pp. 423-425.

Ewbank, M. P. and Andrews, T. J. (2008) 'Differential sensitivity for viewpoint between familiar and unfamiliar faces in human visual cortex', NeuroImage, 40(4), pp. 18571870.

Fellowes, J. M., Remez, R. E. and Rubin, P. E. (1997) 'Perceiving the sex and identity of a talker without natural vocal timbre', Perception and Psychophysics, 59(6), pp. 839849.

Fox, C. J., Iaria, G. and Barton, J. J. S. (2009) 'Defining the face processing network: Optimization of the functional localizer in fMRI.', Human Brain Mapping, 30(5), pp. 1637-1651. doi: 10.1002/hbm.20630.

Friston, K. J. (1994) 'Functional and effective connectivity in neuroimaging: A synthesis', Human Brain Mapping, 2(1-2), pp. 56-78. doi: 10.1002/hbm.460020107.

Friston, K. J. et al. (1997) 'Psychophysiological and modulatory interactions in 
neuroimaging', NeuroImage, 6(3), pp. 218-229. doi: 10.1006/nimg.1997.0291.

Friston, K. J. et al. (eds) (2007) Statistical parametric mapping: the analysis of functional brain images, Academic Press.

Ghazanfar, A. A. et al. (2007) 'Vocal-Tract Resonances as Indexical Cues in Rhesus Monkeys', Current Biology. Elsevier Ltd, 17(5), pp. 425-430. doi: 10.1016/j.cub.2007.01.029.

Girges, C., O’Brien, J. and Spencer, J. (2016) 'Neural correlates of facial motion perception', Social Neuroscience, 11(3), pp. 311-316.

Girges, C., Spencer, J. and O’Brien, J. (2015) 'Categorizing identity from facial motion', The Quarterly Journal of Experimental Psychology. Taylor \& Francis, 68(April 2015), pp. 1832-1843. doi: 10.1080/17470218.2014.993664.

Grill-Spector, K., Knouf, N. and Kanwisher, N. (2004) 'The fusiform face area subserves face perception, not generic within-category identification.', Nature Neuroscience, 7(5), pp. 555-562. doi: 10.1038/nn1224.

He, L. and Dellwo, V. (2016) 'The role of syllable intensity in between-speaker rhythmic variability', International Journal of Speech, Language and the Law, 23(2), pp. 243273. doi: 10.1558/ijsll.v23i2.30345.

Hölig, C. et al. (2014a) 'Brain systems mediating voice identity processing in blind humans', Human Brain Mapping, 35(9), pp. 4607-4619. doi: 10.1002/hbm.22498.

Hölig, C. et al. (2014b) 'Crossmodal plasticity in the fusiform gyrus of late blind individuals during voice recognition', NeuroImage, 103, pp. 374-382. doi: 10.1016/ j.neuroimage.2014.09.050.

Holm, S. (1979) 'A Simple Sequentially Rejective Multiple Test Procedure', Scandinavian Journal of Statistics, 6(2), pp. 65-70.

Ingram, J. C. L., Prandolini, R. and Ong, S. (1996) 'Formant trajectories as indices of phonetic variation for speaker identification', International Journal of Speech Language and the Law. Equinox Publishing, 3(1), pp. 129-145. doi: 10.1558/ijsll.v3i1.129.

Ives, D. T., Smith, D. R. and Patterson, R. D. (2005) 'Discrimination of speaker size from syllable phrases', The Journal of the Acoustical Society of America. 2006/01/20, 118(6), pp. 3816-3822. Available at: http://www.ncbi.nlm.nih.gov/pubmed/16419826.

Jenkins, R. E. et al. (2020) 'Are super-face-recognisers also super-voice-recognisers? Evidence from cross-modal identification tasks', PsyArXiv. doi: 10.31234/osf.io/7xdp3.

Kamachi, M. et al. (2003) “Putting the face to the voice": matching identity across modality', Curr.Biol., 13(19), pp. 1709-1714.

Kanwisher, N., McDermott, J. and Chun, M. M. (1997) 'The fusiform face area: A module in 
human extrastriate cortex specalized for face perception', The Journal of Neuroscience, 17(11), pp. 4302-4311.

Kanwisher, N. and Yovel, G. (2006) 'The fusiform face area: a cortical region specialized for the perception of faces', Philosophical Transactions of the Royal Society B, 361(1476), pp. 2109-2128. doi: 10.1098/rstb.2006.1934.

Kiebel, S. J., Daunizeau, J. and Friston, K. J. (2009) 'Perception and hierarchical dynamics', Frontiers in Neuroinformatics, 3, p. 20. Available at: http://www.ncbi.nlm.nih.gov/entrez/query.fcgi?cmd=Retrieve\&db=PubMed\&dop $\mathrm{t}=$ Citation\&list_uids=19649171.

Kim, C. et al. (2019) 'On learning associations of faces and voices', in C., J. et al. (eds) Computer Vision - ACCV 2018. ACCV 2018. Lecture Notes in Computer Science. vol 11365. Springer, Cham, pp. 276-292. doi: 10.1007/978-3-030-20873-8_18.

Knappmeyer, B., Thornton, I. M. and Bülthoff, H. H. (2003) 'The use of facial motion and facial form during the processing of identity', Vision Research, 43(18), pp. 1921-1936. doi: 10.1016/S0042-6989(03)00236-0.

Knight, B. and Johnston, A. (1997) 'The role of movement in face recognition', Visual Cognition, 4(3), pp. 265-273. doi: 10.1080/713756764.

Krauss, R. M., Freyberg, R. and Morsella, E. (2002) 'Inferring speakers' physical attributes from their voices', Journal of Experimental Social Psychology, 38, pp. 618-625.

von Kriegstein, K. et al. (2003) 'Modulation of neural responses to speech by directing attention to voices or verbal content.', Cognitive Brain Research, 17(1), pp. 48-55.

von Kriegstein, K. et al. (2005) 'Interaction of face and voice areas during speaker recognition', Journal of Cognitive Neuroscience, 17(3), pp. 367-376. doi: 10.1162/0898929053279577.

von Kriegstein, K. et al. (2008) 'Simulation of talking faces in the human brain improves auditory speech recognition.', Proceedings of the National Academy of Sciences, 105(18), pp. 6747-6752. doi: 10.1073/pnas.0710826105.

von Kriegstein, K. (2012) 'A multisensory perspective on human communication', in Murray, M. M. and Wallace, M. T. (eds) The Neural Bases of Multisensory Processes. CRC Press/Taylor \& Francis.

von Kriegstein, K. and Giraud, A.-L. (2006) 'Implicit multisensory associations influence voice recognition', PLoS Biology, 4(10), p. e326. doi: 10.1371/journal.pbio.0040326.

von Kriegstein, K. and Giraud, A. L. (2004) 'Distinct functional substrates along the right superior temporal sulcus for the processing of voices', NeuroImage, 22(2), pp. 948955. doi: 10.1016/j.neuroimage.2004.02.020.

von Kriegstein, K., Kleinschmidt, A. and Giraud, A.-L. (2006) 'Voice recognition and cross- 
modal responses to familiar speakers' voices in prosopagnosia.', Cerebral Cortex, 16(9), pp. 1314-1322. doi: 10.1093/cercor/bhj073.

Lachs, L. and Pisoni, D. B. (2004) 'Cross-modal source information and spoken word recognition', Journal of Experimental Psychology. Human Perception and Performance, 30(2), pp. 378-396. doi: 10.1037/0096-1523.30.2.378.Cross-Modal.

Van Lancker, D., Kreiman, J. and Emmorey, K. (1985) 'Familiar Voice Recognition: Patterns and Parameters. Part I: Recognition of Backward Voices', Journal of Phonetics, 13(1), pp. 19-38.

Van Lancker, D., Kreiman, J. and Wickens, T.-D. (1985) 'Familiar Voice Recognition: Patterns and Parameters. Part II: Recognition of Rate-Altered Voices', Journal of Phonetics, 13(1), pp. 39-52.

Lander, K. and Bruce, V. (2000) 'Recognizing famous faces: Exploring the benefits of facial motion', Ecological Psychology, 12(4), pp. 259-272.

Lander, K., Christie, F. and Bruce, V. (1999) 'The role of movement in the recognition of famous faces', Memory \& Cognition, 27(6), pp. 974-985. doi: 10.3758/BF03201228.

Lander, K. and Chuang, L. (2005) 'Why are moving faces easier to recognize?', Visual Cognition, 12(3), pp. 429-442. doi: 10.1080/13506280444000382.

Latinus, M. and Belin, P. (2011) 'Human voice perception.', Current Biology. Elsevier, 21(4), pp. R143-5. doi: 10.1016/j.cub.2010.12.033.

Lavan, N. et al. (2019) 'Flexible voices: Identity perception from variable vocal signals', Psychonomic Bulletin and Review. Psychonomic Bulletin \& Review, 26(1), pp. 90102. doi: 10.3758/s13423-018-1497-7.

Lavner, Y., Rosenhouse, J. and Gath, I. (2001) 'The prototype model in speaker identification by human listeners', International Journal of Speech Technology, 4(1), pp. 63-74. doi: 10.1023/A:1009656816383.

Leemann, A., Kolly, M. J. and Dellwo, V. (2014) 'Speaker-individuality in suprasegmental temporal features: Implications for forensic voice comparison', Forensic Science International. Elsevier Ireland Ltd, 238, pp. 59-67. doi: 10.1016/j.forsciint.2014.02.019.

Liu, J., Harris, A. and Kanwisher, N. (2010) 'Perception of face parts and face configurations: An FMRI study', Journal of Cognitive Neuroscience, 22(1), pp. 203-211.

Liu, R. R. et al. (2015) 'The processing of voice identity in developmental prosopagnosia', Cortex. Elsevier Ltd, 71, pp. 390-397. doi: 10.1016/j.cortex.2015.07.030.

Longmore, C. A. and Tree, J. J. (2013) 'Motion as a cue to face recognition: Evidence from congenital prosopagnosia', Neuropsychologia. Elsevier, 51(5), pp. 864-875. doi: 10.1016/j.neuropsychologia.2013.01.022. 
Luzzi, S. et al. (2018) 'Selective associative phonagnosia after right anterior temporal stroke', Neuropsychologia, 31(116), pp. 154-161. doi: 10.1016/j.neuropsychologia.2017.05.016.

Maguinness, C. and von Kriegstein, K. (2017) 'Cross-modal processing of voices and faces in developmental prosopagnosia and developmental phonagnosia', Visual Cognition. Taylor \& Francis, 25(4-6), pp. 644-657. doi: 10.1080/13506285.2017.1313347.

Maguinness, C. and Newell, F. N. (2015) 'Non-rigid, but not rigid, motion interferes with the processing of structural face information in developmental prosopagnosia', Neuropsychologia. Elsevier, 70, pp. 281-295. doi: 10.1016/j.neuropsychologia.2015.02.038.

Maguinness, C., Roswandowitz, C. and von Kriegstein, K. (2018) 'Understanding the mechanisms of familiar voice-identity recognition in the human brain', Neuropsychologia. Elsevier Ltd, 116, pp. 179-193. doi: 10.1016/j.neuropsychologia.2018.03.039.

Maguinness, C., Schall, S. and von Kriegstein, K. (2021) 'Prior audio-visual learning facilitates auditory-only speech and voice-identity recognition in noisy listening conditions', PsyArXiv. doi: https://doi.org/10.31234/osf.io/gc4xa.

Mavica, L. W. and Barenholtz, E. (2013) 'Matching voice and face identity from static images.', Journal of Experimental Psychology: Human Perception and Performance, 39(2), pp. 307-312. doi: 10.1037/a0030945.

Mc Dougall, K. (2004) 'Speaker-specific formany dynamics: An experiment on Australian English /ai/', Speech, Language and the Law, 11(1), pp. 103-130. doi: 10.1097/PSY.0000000000000183.

Mc Dougall, K. (2006) 'Dynamic features of speech and the characterization of speakers: towards a new approach using formant frequencies', Speech, Language and the Law, 13(1), pp. 89-126. doi: 10.1097/PSY.0000000000000183.

Mc Dougall, K. and Nolan, F. (2007) 'Discrimination of speakers using the formant dynamics of /u:/ in British English', Proceedings of the 16th International Congress of Phonetic Sciences, pp. 1825-1828. Available at: http://www.icphs2007.de.

McConachie, H. R. (1976) 'Developmental prosopagnosia. A single case report', Cortex, 12(1), pp. 76-82. doi: 10.1016/S0010-9452(76)80033-0.

Nichols, T. and Hayasaka, S. (2003) 'Controlling the familywise error rate in functional neuroimaging: a comparative review', Statistical Methods in Medical Research, 12, pp. 419-446.

O'Reilly, J. X. et al. (2012) 'Tools of the trade: psychophysiological interactions and functional connectivity.', Social Cognitive and Affective Neuroscience, 7(5), pp. 604609. 
O’Toole, A. J., Roark, D. A. and Abdi, H. (2002) 'Recognizing moving faces: A psychological and neural synthesis', Trends in Cognitive Sciences, 6(6), pp. 261-266. doi: 10.1016/S1364-6613(02)01908-3.

Oh, T. H. et al. (2019) 'Speech2Face: Learning the face behind a voice', in Proceedings of the IEEE Computer Society Conference on Computer Vision and Pattern Recognition. IEEE Computer Society, pp. 7531-7540. doi: 10.1109/CVPR.2019.00772.

Oldfield, R. C. (1971) 'The assessment and analysis of handedness: The edinburgh inventory', Neuropsycholgia, 9, pp. 97-113.

Pernet, C. R. and Belin, P. (2012) 'The role of pitch and timbre in voice gender categorization.', Frontiers in psychology, 3(February), p. 23. doi: 10.3389/fpsyg.2012.00023.

Pitcher, D. et al. (2011) 'Differential selectivity for dynamic versus static information in faceselective cortical regions.', NeuroImage, 56(4), pp. 2356-2363. doi: 10.1016/j.neuroimage.2011.03.067.

Remez, R. E., Fellowes, J. M. and Rubin, P. E. (1997) 'Talker identification based on phonetic information', Journal of Experimental Psychology: Human Perception and Performance, 23(3), pp. 651-666.

De Renzi, E. (1986) 'Prosopagnosia in two patients with CT scan evidence of damage confined to the right hemisphere', Neuropsychologia, 24(3), pp. 385-389.

Richardson, J. T. E. (2011) 'Eta squared and partial eta squared as measures of effect size in educational research', Educational Research Review. Elsevier Ltd, 6(2), pp. 135-147. doi: 10.1016/j.edurev.2010.12.001.

Riedel, P. et al. (2015) 'Visual face-movement sensitive cortex is relevant for auditory-only speech recognition', Cortex, 68, pp. 86-99. doi: 10.1016/j.cortex.2014.11.016.

Roark, D. A. et al. (2003) 'Memory for moving faces: Psychological and neural perspectives on the role of motion in face recognition', Behavioral and Cognitive Neuroscience Reviews, 2(1), pp. 15-46.

Rosenblum, L. D., Johnson, J. A. and Saldana, H. M. (1996) 'Visual kinematic information for embellishing speech in noise', Journal of Speech and Hearing Research, 39(6), pp. 1159-1170.

Ross, L. a et al. (2007) 'Do you see what I am saying? Exploring visual enhancement of speech comprehension in noisy environments.', Cerebral Cortex, 17(5), pp. 11471153. doi: $10.1093 /$ cercor/bhl024.

Rossion, B. (2014) 'Understanding face perception by means of prosopagnosia and neuroimaging.', Frontiers in Bioscience, 6, pp. 258-307.

Rotshtein, P. et al. (2005) 'Morphing Marilyn into Maggie dissociates physical and identity 
face representations in the brain.', Nature neuroscience, 8(1), pp. 107-113. doi: $10.1038 / \mathrm{nn} 1370$.

Schall, S. et al. (2013) 'Early auditory sensory processing of voices is facilitated by visual mechanisms', NeuroImage. Elsevier Inc., 77, pp. 237-245. doi: 10.1016/j.neuroimage.2013.03.043.

Schall, S. et al. (2014) 'Voice identity recognition: Functional division of the right STS and its behavioural relevance', Journal of Cognitive Neuroscience, 27(2), pp. 280-291.

Schall, S. and von Kriegstein, K. (2014) 'Functional connectivity between face-movement and speech-intelligibility areas during auditory-only speech perception.', PLOS ONE, 9(1), p. e86325. doi: 10.1371/journal.pone.0086325.

Schelinski, S., Borowiak, K. and von Kriegstein, K. (2016) 'Temporal voice areas exist in autism spectrum disorder but are dysfunctional for voice identity recognition', Social Cognitive and Affective Neuroscience, 11(11), pp. 1812-1822. doi: 10.1093/scan/nsw089.

Schelinski, S., Riedel, P. and von Kriegstein, K. (2014) 'Visual abiltites are important for auditory-only speech recognition: Evidence from autism spectrum disorder', Neuropsychologia, 65, pp. 1-11.

Schiltz, C. et al. (2010) 'Holistic perception of individual faces in the right middle fusiform gyrus as evidenced by the composite face illusion', Journal of Vision. The Association for Research in Vision and Ophthalmology, 10(2), pp. 1-16. doi: 10.1167/10.2.25.

Schultz, J. and Pilz, K. S. (2009) 'Natural facial motion enhances cortical responses to faces.', Experimental Brain Research., 194(3), pp. 465-475. doi: 10.1007/s00221-0091721-9.

Schweinberger, S. R. (2001) 'Human brain potential correlates of voice priming and voice recognition.', Neuropsychologia, 39(9), pp. 921-936. Available at: http://www.ncbi.nlm.nih.gov/pubmed/11516445.

Schweinberger, S. R., Kloth, N. and Robertson, D. M. C. (2011) 'Hearing facial identities: brain correlates of face-voice integration in person identification', Cortex. Elsevier Srl, 47(9), pp. 1026-1037. doi: 10.1016/j.cortex.2010.11.011.

Schweinberger, S. R. and Robertson, D. M. C. (2017) 'Audiovisual integration in familiar person recognition', Visual Cognition. Taylor \& Francis, 0(0), pp. 1-22. doi: $10.1080 / 13506285.2016 .1276110$.

Shams, L. and Seitz, A. R. (2008) 'Benefits of multisensory learning.', Trends in cognitive sciences, 12(11), pp. 411-7. doi: 10.1016/j.tics.2008.07.006.

Sheffert, S. M. et al. (2002) 'Learning to recognize talkers from natural, sinewave, and reversed speech samples.', Journal of experimental psychology. Human perception and performance, 28(6), pp. 1447-1469. doi: 10.1037/0096-1523.28.6.1447. 
Sheffert, S. M. and Olson, E. (2004) 'Audiovisual speech facilitates voice learning.', Perception \& Psychophysics, 66(2), pp. 352-362. Available at: http://www.ncbi.nlm.nih.gov/pubmed/15129754.

Sidtis, D. and Kreiman, J. (2012) 'In the beginning was the familiar voice: personally familiar voices in the evolutionary and contemporary biology of communication', Integr Psychol Behav Sci, 46(2), pp. 146-159.

Simmons, D. et al. (2020) 'Cross-modal transfer of talker-identity learning', Attention, Perception \& Psychophysics. Attention, Perception, \& Psychophysics. doi: https://doi.org/10.3758/s13414-020-02141-9.

Smith, D. R. R. et al. (2005) 'The processing and perception of size information in speech sounds', J.Acoust.Soc.Am., 117(1), pp. 305-318.

Smith, D. R. R. and Patterson, R. D. (2005) 'The interaction of glottal-pulse rate and vocaltract length in judgements of speaker size, sex and age', Journal of the Acoustical Society of America, 118, pp. 3177-3186.

Smith, H. M. J. et al. (2016) 'Concordant Cues in Faces and Voices: Testing the Backup Signal Hypothesis', Evolutionary Psychology, 14(1), pp. 1-10. doi: $10.1177 / 1474704916630317$.

Smith, H M J et al. (2016) 'Matching novel face and voice identity using static and dynamic facial images', Attention, Perception, \& Psychophysics, 78(3), pp. 868-879. doi: 10.3758/s13414-015-1045-8.

Steede, L. L., Tree, J. J. and Hole, G. J. (2007) 'I can't recognize your face but I can recognize its movement.', Cognitive Neuropsychology, 24(4), pp. 451-466. doi: 10.1080/02643290701381879.

Stevenage, S. V. (2018) 'Drawing a distinction between familiar and unfamiliar voice processing: A review of neuropsychological, clinical and empirical findings', Neuropsychologia. Elsevier Ltd, 116, pp. 162-178. doi: 10.1016/j.neuropsychologia.2017.07.005.

Sumby, W. H. and Pollack, I. (1954) 'Visual contribution of speech intelligibility in noise', The Journal of the Acoustical Society of America, 26(2), pp. 212-215.

Traunmüller, H. and Eriksson, A. (1994) 'The frequency range of the voice fundamental in the speech of male and female adults', Department of Linguistics, University of Stockholm, 97, pp. 1905191-1905195.

Tsantani, M. et al. (2019) 'Faces and voices in the brain: A modality-general person-identity representation in superior temporal sulcus', NeuroImage. Elsevier Ltd, 201, p. 116004. doi: 10.1016/j.neuroimage.2019.07.017. 
Voiers, W. D. (1964) 'Perceptual Bases of Speaker Identity', The Journal of the Acoustical Society of America, 36(6), pp. 1065-1073.

Watson, R. et al. (2014) 'People-selectivity, audiovisual integration and heteromodality in the superior temporal sulcus.', Cortex. Elsevier Ltd, 50, pp. 125-136. doi: 10.1016/j.cortex.2013.07.011.

Weibert, K. and Andrews, T. J. (2015) 'Activity in the right fusiform face area predicts the behavioural advantage for the perception of familiar faces', Neuropsychologia. Elsevier, 75, pp. 588-596. doi: 10.1016/j.neuropsychologia.2015.07.015.

$\mathrm{Xu}, \mathrm{X}$. et al. (2009) 'Adaptation in the fusiform face area (FFA): Image or person?', Vision Research. Elsevier Ltd, 49(23), pp. 2800-2807. doi: 10.1016/j.visres.2009.08.021.

Young, A. W., Frühholz, S. and Schweinberger, S. R. (2020) 'Face and voice perception: Understanding commonalities and differences', Trends in Cognitive Sciences. Elsevier Ltd, 24(5), pp. 398-410. doi: 10.1016/j.tics.2020.02.001.

Yovel, G. and O'Toole, A. J. (2016) 'Recognizing People in Motion', Trends in Cognitive Sciences. Elsevier Ltd, 20(5), pp. 383-395. doi: 10.1016/j.tics.2016.02.005.

Zäske, R., Mühl, C. and Schweinberger, S. R. (2015) 'Benefits for voice learning caused by concurrent faces develop over time', PLoS ONE, 10(11), pp. 1-12. doi: 10.1371/journal.pone.0143151.

Zuo, D. and Mok, P. P. K. (2015) 'Formant dynamics of bilingual identical twins', Journal of Phonetics. Elsevier, 52, pp. 1-12. doi: 10.1016/j.wocn.2015.03.003.

\section{Acknowledgements}

We thank Kamila Borowiak for comments on an earlier version of this manuscript and Stefanie Schelinski for providing the visual face area localiser experiment. This work was funded by a Max Planck Research Group grant to KvK. CM also receives funding from the ERC-consolidator grant SENSOCOM 647051 and the DFG KR 3735/5-1.

\section{Conflict of Interest}

The authors declare no competing financial interests.

\section{Author Contributions}

$\mathrm{CM}$ and KvK designed the research; CM performed the research and data analysis; CM and KvK wrote the article. 


\section{Supporting Information}

Supporting Figures and Tables Referenced in the Main Manuscript

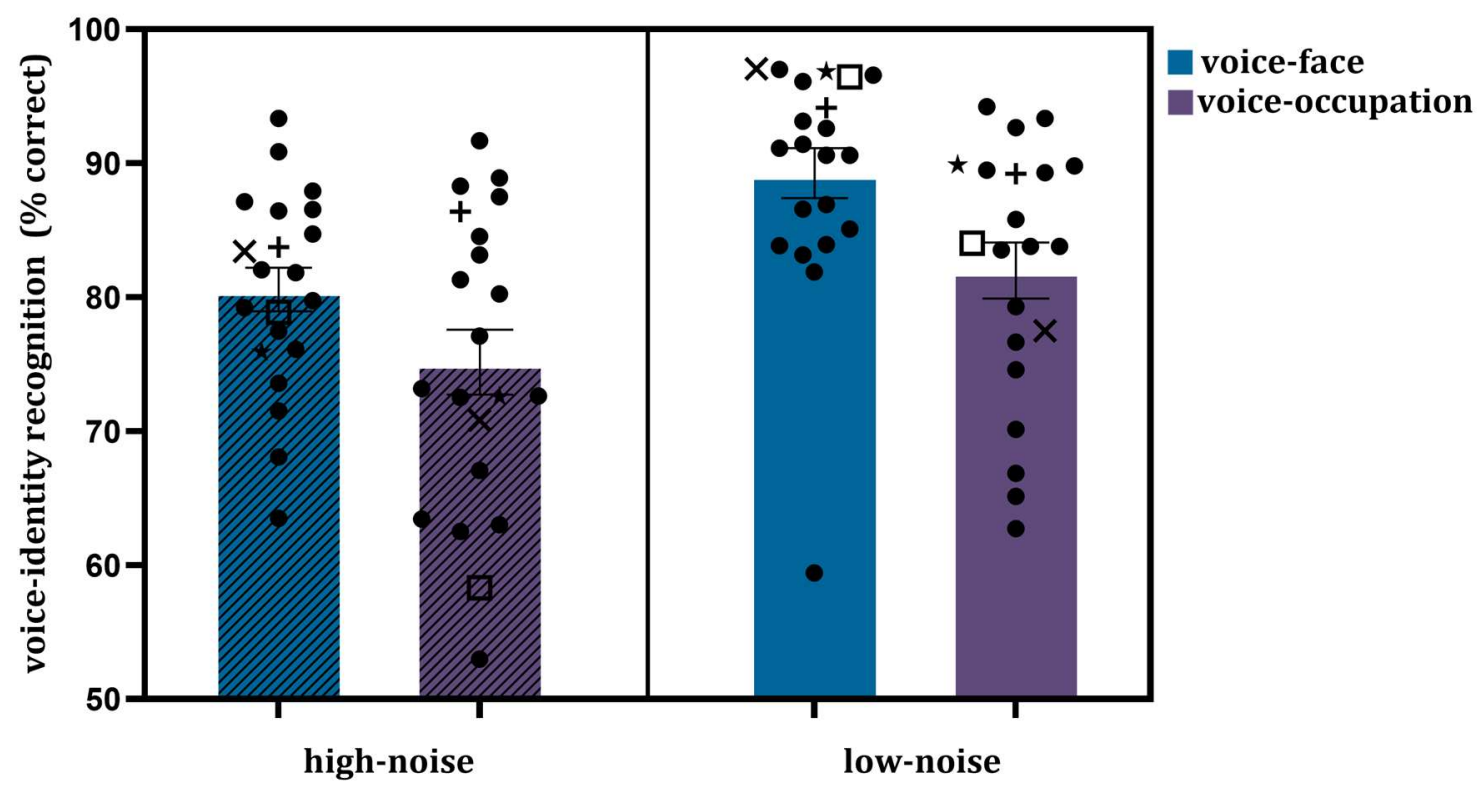

Supp. Figure 1. Auditory-only voice-identity recognition accuracy scores. Accuracy scores are displayed for speakers learned by face or occupation, in high- and low-noise listening conditions. Bar plots display the mean accuracy scores for $\mathrm{N}=21$. Error bars denote $+/-1$ standard error of the mean. Individual participant scores are displayed as single points. The 4 participants who completed the high-noise listening condition with SNR $-8 \mathrm{~dB}$ are each displayed with a unique symbol per participant $(\square ;+; \times ; \star)$ to facilitate comparison to all collected data. All other individuals, displayed as circular points, were presented with audio stimuli with an SNR - $4 \mathrm{~dB}$ for the high-noise listening condition. 


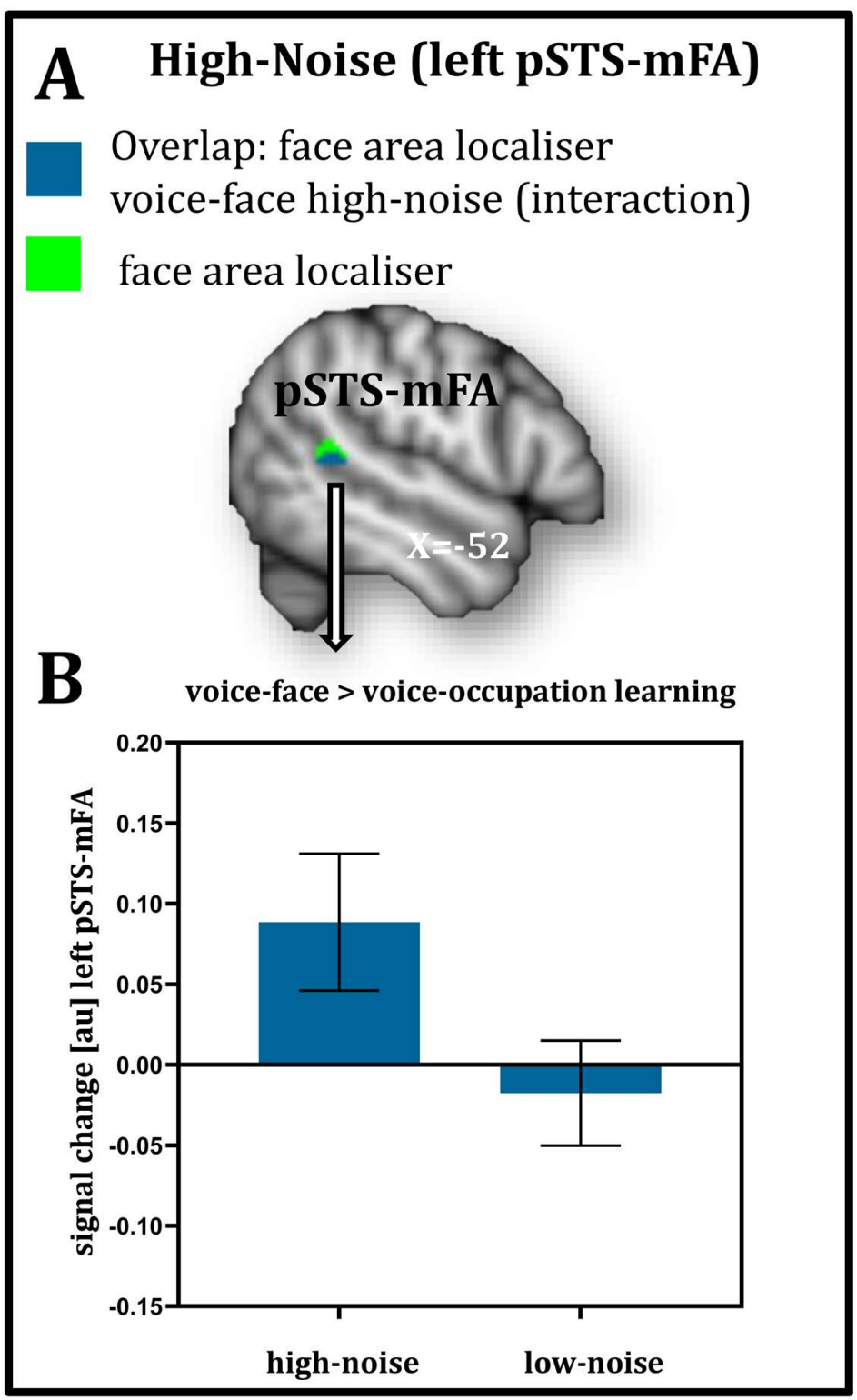

Supp. Figure 2. fMRI results. Overview of responses in the left pSTS-mFA during voice-identity recognition for face-learned speakers in high-noise. A. Overlay of the interaction contrast '(voice-face high-noise $>$ voice-occupation high-noise) > (voice-face low-noise > voiceoccupation low-noise)' and the functional face-area localiser contrast for the left pSTS-mFA ('dynamic faces > dynamic objects'). B. Signal change estimate in the left pSTS-mFA for the conditions included in the interaction contrast '(voice-face high-noise > voice-occupation high-noise) > (voice-face lownoise > voice-occupation lownoise)' during auditory-only voice-identity recognition. Plot shows the first eigenvariate extracted from the maximum statistic for the interaction contrast shown in $\mathrm{A} ; \mathrm{x}=-54, \mathrm{y}=$ $-43, \mathrm{z}=8, p=0.027$, FWE corrected for ROI. The interaction in the left pSTS-mFA did not survive Holm-Bonferroni correction for the 3 ROIs. In (A) overlapping responses between the voice-identity recognition contrast and the face-area localiser contrast are shown in blue (for display purposes presented at $p=0.05$ whole brain uncorrected, masked by the ROI). Non-overlapping responses in the functionally localised left pSTS-mFA are displayed in green. All responses are overlaid on a mean structural MNI152 T1 weighted image (sagittal view, left hemisphere) and visualised using MRIcron (www.nitrc.org/projects/mricron). All co-ordinates are reported in MNI-space. 
Supp. Table 1. Group MNI co-ordinates for the functionally localised face areas for $\mathrm{N}=20$ and $\mathrm{N}=$ 15 (face-benefit) participants.

\begin{tabular}{l|ccccc|ccccc}
\hline \multicolumn{10}{c}{ Face area ROIs } \\
\hline & $\mathrm{x}$ & $\mathrm{y}$ & $\mathrm{z}$ & $\begin{array}{c}\text { cluster size } \\
\text { (voxels) }\end{array}$ & $\begin{array}{c}T- \\
\text { value }\end{array}$ & $\mathrm{x}$ & $\mathrm{y}$ & $\mathrm{z}$ & $\begin{array}{c}\text { cluster size } \\
\text { (voxels) }\end{array}$ & $\begin{array}{c}T- \\
\text { value }\end{array}$ \\
\hline \multicolumn{1}{l}{ All Participants $(\mathrm{N}=20)$} \\
\hline FFA & 45 & -40 & -19 & 25 & 3.54 & 45 & -37 & -19 & 25 & 3.14 \\
Right pSTS-mFA & 54 & -34 & 2 & 29 & 4.55 & 54 & -31 & 2 & 30 & 3.88 \\
Left pSTS-mFA & -51 & -46 & 11 & 29 & 3.89 & -51 & -46 & 11 & 30 & 3.89 \\
\hline
\end{tabular}

\section{Supporting Functional MRI Analysis}

\section{Main Effect of Noise-Level}

We examined if there were increased responses in the visual ROIs during the recognition of speakers in high-noise, in comparison to low noise listening conditions, regardless of the audio-visual condition that the speakers were learned through (i.e., voiceface or voice-occupation). To do this, we used the main effect of noise-level contrast (highnoise > low-noise). This analysis showed no increased responses in any of the visual ROIs i.e., FFA, left pSTS-mFA, or right pSTS-mFA, even at lenient thresholds of $p<0.05$ uncorrected. This was observed for the analysis including all participants $(\mathrm{N}=21)$ and for the 16 participants who had a positive face-benefit score. 